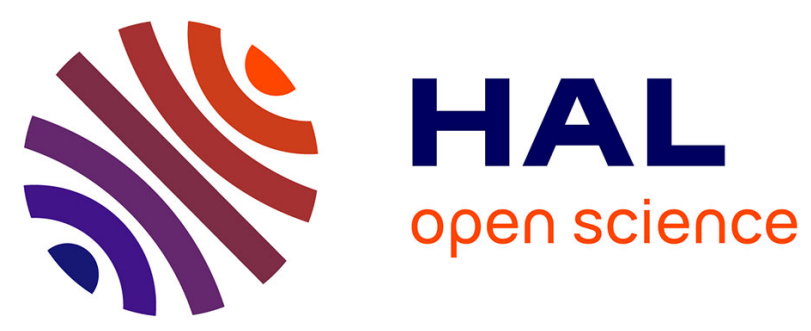

\title{
NUMERICAL ANALYSIS OF THE TOPOLOGICAL GRADIENT METHOD FOR FOURTH ORDER MODELS AND APPLICATIONS TO THE DETECTION OF FINE STRUCTURES IN IMAGING
}

\author{
Audric Drogoul
}

\section{To cite this version:}

Audric Drogoul. NUMERICAL ANALYSIS OF THE TOPOLOGICAL GRADIENT METHOD FOR FOURTH ORDER MODELS AND APPLICATIONS TO THE DETECTION OF FINE STRUCTURES IN IMAGING. 2014. hal-01018204v1

\author{
HAL Id: hal-01018204 \\ https://hal.science/hal-01018204v1
}

Preprint submitted on 3 Jul 2014 (v1), last revised 7 Jan 2015 (v2)

HAL is a multi-disciplinary open access archive for the deposit and dissemination of scientific research documents, whether they are published or not. The documents may come from teaching and research institutions in France or abroad, or from public or private research centers.
L'archive ouverte pluridisciplinaire HAL, est destinée au dépôt et à la diffusion de documents scientifiques de niveau recherche, publiés ou non, émanant des établissements d'enseignement et de recherche français ou étrangers, des laboratoires publics ou privés. 


\title{
NUMERICAL ANALYSIS OF THE TOPOLOGICAL GRADIENT METHOD FOR FOURTH ORDER MODELS AND APPLICATIONS TO THE DETECTION OF FINE STRUCTURES IN IMAGING
}

\author{
AUDRIC DROGOUL ${ }^{\dagger}$
}

\begin{abstract}
In this paper we present the numerical analysis of the topological gradient method developed in [13] for the detection of fine structures (filaments and points in 2D). First used in mechanics of structures [28], this method has been then applied in imaging for edge detection and image restoration $[8,14]$. The model involves second order derivatives and leads to fourth order PDEs. We first develop the case of Gaussian noisy images and then we extend the method to the more general case of blurred and Gaussian noisy images. We show that as for edge detection [14], the topological gradient is not only a filament detector but it also allows to restore images containing filaments. Then we extend the approach to surfaces and filaments detection in 3D. We experiment all of the presented methods on synthetic and real images and compare our results with some classical methods.
\end{abstract}

Key words. Fine structures, Topological gradient, Segmentation, Restoration

AMS subject classifications. 35J30, 49Q10, 49Q12, 94A08, 94A13

1. Introduction. The main goal of this paper is to propose a topological gradient based method for the detection of fine structures in 2D (filaments and points). This article is a companion paper of [13] and more precisely it is its numerical and applied version. Before presenting the model, let us recall the notion of topological gradient and the problem of detection of fine structures in $2 \mathrm{D}$ images. The topological sensitivity analysis is the study of the variations of a cost function with respect to a topological modification of an open domain of $\mathbb{R}^{2}$, such as the creation of a hole or a crack. In order to fix ideas, let $\Omega \subset \mathbb{R}^{2}$ be the image domain, of boundary $\partial \Omega=\Gamma$, and let $j(\Omega)=J\left(\Omega, u_{\Omega}\right)$ be a cost function where $u_{\Omega}$ is the solution of a partial differential equation defined on $\Omega$. For small $\epsilon>0$, let (a) $\Omega_{\epsilon}=\Omega \backslash \overline{\left\{x_{0}+\epsilon \omega\right\}}$ or (b) $\Omega_{\epsilon}=\Omega \backslash \overline{\left\{x_{0}+\epsilon \sigma(\vec{n})\right\}}$ be the perturbed domain, where $x_{0} \in \Omega, \omega=B(O, 1)$ is the unit ball of $\mathbb{R}^{2}$ and $\sigma(\vec{n})$ is a straight segment (crack) with normal $\vec{n}$ (see Figure 1).

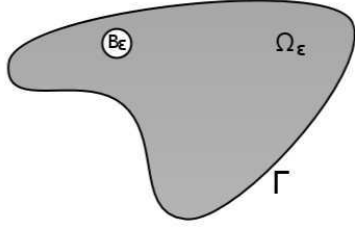

(a) Perforated domain

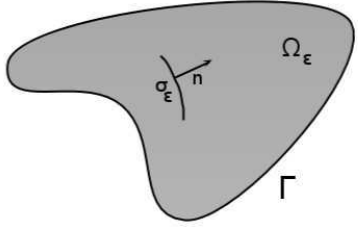

(b) Cracked domain

FIG. 1.

Generally in 2D and for Neumann problems, we have the following expansion with respect to $\epsilon: j\left(\Omega_{\epsilon}\right)=j(\Omega)+\epsilon^{2} \mathcal{I}\left(x_{0}\right)+o\left(\epsilon^{2}\right)$ where $\mathcal{I}\left(x_{0}\right)$ is the topological gradient defined by $\mathcal{I}\left(x_{0}\right)=\lim _{\epsilon \rightarrow 0} \frac{j\left(\Omega_{\epsilon}\right)-j(\Omega)}{\epsilon^{2}}$. Thus if we want to minimize $j\left(\Omega_{\epsilon}\right)$ it would be preferable to create holes or cracks at points $x_{0}$ where $\mathcal{I}\left(x_{0}\right)$ is "the most

\footnotetext{
${ }^{\dagger}$ drogoula@unice.fr

Univ. Nice Sophia Antipolis, CNRS, LJAD, UMR 7351, 06100 Nice, France
} 
negative". Initially defined in $[29,21]$ and used in mechanics for crack detection [28] or engineering design, the notion of topological gradient has been recently applied to imaging problems such as restoration/segmentation or classification of images ([4, $8,5])$. For example in restauration/segmentation, if $f$ denotes the observed image (possibly noised and/or blurred) we look for a couple $(u, \gamma)$, where $\gamma$ is the set of edges of the image and $\mathrm{u}$ is a regularized version of $f$ on $\Omega \backslash \gamma$. Many variational methods have been proposed in the literature ([3]) and the most famous one is the Mumford and Shah [11] model. A recent alternative was the introduction of the topological gradient method. For edge detection the simpler model $[4,8,5]$ is based on the Laplace operator. The associated energy uses the spatial gradient of $u_{\Omega}$ : $J\left(\Omega, u_{\Omega}\right)=\int_{\Omega}\left|\nabla u_{\Omega}\right|^{2} d x$. The main difference between edges and filaments is that for edges the singularity is associated with a jump of the intensity across edges while for filaments there is no jump (see Figure 2). If for edge detection the usual spatial

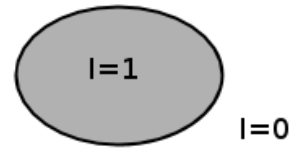

(a)

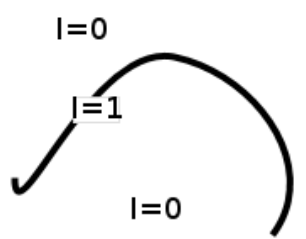

(b)

FIG. 2. (a): An edge with a jump of the intensity I across the boundary of the object; (b) A filament without jump across it.

gradient is classically used, it is inefficient for the detection of points or filaments. To illustrate this fact let us consider in 1D the function $f(x)=0$ if $x \neq 0$ and $f(0)=1$. This function can be approximated by the function $f_{\eta}(x)=0$ if $|x| \geq \eta$ and $f_{\eta}(x)=\frac{2}{\eta^{3}}|x|^{3}-\frac{3}{\eta^{2}}|x|^{2}+1$ if $|x| \leq \eta$. We have $f_{\eta}^{\prime}(0)=0$ but $f_{\eta}^{\prime \prime}(0)=\frac{-6}{\eta^{2}}$, thus $f_{\eta}^{\prime}$ "does not see" 0 but $f_{\eta}^{\prime \prime}$ becomes singular at 0 . In fact, it is known $([30,24])$ that if we want to detect fine structures by using differential operators, we must use at least second order operators. For these reasons, in [13], we have chosen a variational model using second order derivatives. We will describe this model in section 2. Other variational models have been proposed in the literature according to applications, see [20] for the detection of biological filaments or [26] for road network detection. In $[2,7]$ and $[6]$ the authors propose a model for detecting objects of codimension two and one in 2D images. Their method is inspired by Ginzburg-Landau models. In [16] a variational model involving second order differential operators is developed for detecting point-like singularities. There exists of course other approaches not based on variational calculus. In [22, 34] morphological methods are presented. In [34], authors propose a method for automatic detection of vessel-like patterns using morphological filters and curvature evaluation.

The method is sensitive to the noise type (uniform noise destructs small vessels) and the computation time is not negligible. In $[18,33,32]$, stochastic methods are used. Here filaments are defined as the realization of random processes. These methods are costly in terms of CPU time. In [32] a thin network is simulated by a point process which penalizes not connected segments and favors aligned segments. The estimate of the network is obtained by minimising an energy function. Finally let us 
mention the wavelet approach $[1,19,9]$.

The remainder of this paper is organized as follows. In section 2 we present the variational model and we justify thaks to an heuristic reasoning, the choice of the cost function and associated PDE. Then in section 3 we recall the theoretical value of the topological gradient given in [13]. We extend the result to a more general model of image degradation in section 4 . We will see in section 5 that the topological gradient is not only a detector but it also allows to restore images. To compare our indicator with others one, in section 6 we present a classical indicator often used in filaments and points detection [31, 30, 24] . In section 7 we develop the numerical analysis of the topological gradient method. In section 8 and section 9 we give some experiment results on in filaments and points detection for possibly noised images. We numerically justify the pertinence of the model, and compare the results obtained by the topological gradient method with the ones given by the indicator introduced in section 6 . In section 10, we experiment the segmentation given in section 4 for blurred and noisy images and we illustrate the restoration previously presented in section 5 . Finally, in section 11 we give the value of the topological gradient in the $3 \mathrm{D}$ case and we present some numerical results.

2. Definition of the cost function and variational model. In this section we give a lemma explaining why we need a cost function using second order derivatives to detect fine structures in $2 \mathrm{D}$ images and then we set the chosen model defined on $\Omega_{\epsilon}$.

2.1. What is the good operator for detecting fine structures?. We denote by $\mathcal{D}\left(\mathbb{R}^{2}\right)$ the space of $C^{\infty}$-functions with compact support in $\mathbb{R}^{2}$ and $\mathcal{D}^{\prime}\left(\mathbb{R}^{2}\right)$ the space of distributions on $\mathbb{R}^{2}$. For the proof of this lemma we refer to [12].

Lemma 2.1. Let $\varphi: \mathbb{R}^{2} \longrightarrow \mathbb{R}$ a Lipschitz continuous function, and let $\left(g_{h}\right)_{h>0}$ a sequence of functions defined by

$$
g_{h}(x)=\frac{1}{\theta_{1}(h)} e^{-\frac{\varphi^{2}(x)}{\theta_{2}(h)}}
$$

where $\theta_{i}: \mathbb{R}^{+} \longrightarrow \mathbb{R}^{+}$and $\lim _{h \rightarrow 0} \theta_{i}(h)=0$.

(1) Let $a \in \mathbb{R}^{2}, \varphi(x)=\|x-a\|, \theta_{1}(h)=\pi h$ and $\theta_{2}(h)=h$, then

$$
g_{h} \underset{h \rightarrow 0}{\longrightarrow} \delta_{a}, \text { in } \mathcal{D}^{\prime}\left(\mathbb{R}^{2}\right)
$$

Moreover we have

$$
\nabla g_{h}(a)=[0,0]^{T} \quad, \quad \nabla^{2} g_{h}(a)=-\frac{2}{\pi h^{2}} I
$$

where I denotes the identity matrix in $\mathbb{R}^{2}$.

(2) Let $\Gamma$ a smooth closed curve or a smooth infinite curve of $\mathbb{R}^{2}$ delimiting two sub-domains $\mathbb{R}_{\Gamma}^{2-}$ and $\mathbb{R}_{\Gamma}^{2+}$ forming a partition of $\mathbb{R}^{2}$. Let $\varphi$ the signed distance to $\Gamma$ defined by :

$$
\varphi(x)=\operatorname{dist}\left(x, \mathbb{R}_{\Gamma}^{2-}\right)-\operatorname{dist}\left(x, \mathbb{R}_{\Gamma}^{2+}\right)
$$

$\mathbb{R}_{\Gamma}^{2+}$ (resp. $\mathbb{R}_{\Gamma}^{2-}$ ) indentifies to the sub-domain $\{\varphi>0\}$ (resp. $\{\varphi<0\}$ ). Taking the following scalings : $\theta_{1}(h)=\sqrt{\pi h}$ and $\theta_{2}(h)=h$, then we have

$$
g_{h} \underset{h \rightarrow 0}{\longrightarrow} \delta_{\Gamma}, \text { in } \mathcal{D}^{\prime}\left(\mathbb{R}^{2}\right)
$$


Moreover for all $x \in \Gamma$ :

$$
\nabla g_{h}(x)=[0,0]^{T} \quad, \quad \operatorname{spec}\left(\nabla^{2} g_{h}(x)\right)=\left\{-\frac{2}{h^{3 / 2}}, 0\right\}
$$

where $\operatorname{spec}(M)$ denotes the eigenvalues of the matrix $M$. The associated eigenvectors on $\Gamma$ are $\left(\nabla \varphi(x), \nabla \varphi(x)^{\perp}\right)$ where $\nabla \varphi(x)=\vec{n}(x)$ is the unit normal of $\Gamma$.

In 2D images, filaments can be roughly modeled by an indicator function supported by a smooth curve $\Gamma$. By using Lemma 2.1, we can approximate the filament by a sequence of smooth functions, whose Hessian matrices blow up in the perpendicular direction to $\Gamma$ while their gradient are null. Hence this shows that the appropriate operator for detecting such structures must involve second order derivatives of the image we want to recover.

2.2. Definition of the cost function and the fourth order PDE. Now we introduce the cost function and the PDE studied in [13]. The model is inspired by the Kirchhoff thin static plate model subject to pure bending (see [15]) with a Poisson ratio $\nu=0$. The cost function is defined by :

$$
J_{\epsilon}(u)=J_{\Omega_{\epsilon}}(u)=\int_{\Omega_{\epsilon}}\left(\frac{\partial^{2} u}{\partial x_{1}^{2}}\right)^{2}+\left(\frac{\partial^{2} u}{\partial x_{2}^{2}}\right)^{2}+2\left(\frac{\partial^{2} u}{\partial x_{1} \partial x_{2}}\right)^{2}=\int_{\Omega_{\epsilon}}\left\|\nabla^{2} u\right\|^{2},
$$

and $\Omega_{\epsilon}$ is either (a) $\Omega_{\epsilon}=\Omega \backslash \overline{\left\{x_{0}+\epsilon \omega\right\}}$ or (b) $\Omega_{\epsilon}=\Omega \backslash \overline{\left\{x_{0}+\epsilon \sigma(\vec{n})\right\}}$. For a matrix $M$ we denote $\|M\|^{2}=\operatorname{tr}\left(M^{T} M\right)$. Let $u_{\Omega_{\epsilon}}=u_{\epsilon}$ be a regularization of the observed image $f \in L^{2}\left(\Omega_{\epsilon}\right)$ solution of the following minimization problem :

$$
\min _{u \in H^{2}\left(\Omega_{\epsilon}\right)}\left(\alpha J_{\epsilon}(u)+\|u-f\|_{L^{2}\left(\Omega_{\epsilon}\right)}^{2}\right)
$$

where $\alpha>0$ is a parameter to be tuned. The variational formulation of $\left(\mathcal{P}_{\epsilon}\right)$ writes as : find $u_{\epsilon} \in H^{2}\left(\Omega_{\epsilon}\right)$ such that

$$
a_{\epsilon}\left(u_{\epsilon}, v\right)=l_{\epsilon}(v) \quad \forall v \in H^{2}\left(\Omega_{\epsilon}\right)
$$

where $a_{\epsilon}(u, v)$ and $l_{\epsilon}(v)$ denote the bilinear and linear forms:

$$
a_{\epsilon}(u, v)=\int_{\Omega_{\epsilon}} \alpha \sum_{1 \leq i, j \leq 2} \frac{\partial^{2} u}{\partial x_{i} \partial x_{j}} \frac{\partial^{2} v}{\partial x_{i} \partial x_{j}}+u v \quad l_{\epsilon}(v)=\int_{\Omega_{\epsilon}} f v .
$$

The Euler equations associated with $\left(\mathcal{P}_{\epsilon}\right)$ are

$$
\left(\mathcal{P}_{\epsilon}\right)\left\{\begin{aligned}
\alpha \Delta^{2} u_{\epsilon}+u_{\epsilon} & =f \text { on } \Omega_{\epsilon} \\
B_{1}\left(u_{\epsilon}\right) & =0 \text { on } \partial \Omega_{\epsilon} \\
B_{2}\left(u_{\epsilon}\right) & =0 \text { on } \partial \Omega_{\epsilon}
\end{aligned}\right.
$$

where 


$$
\begin{aligned}
& B_{1}(u)=\partial_{n}(\Delta u)-\partial_{\sigma}\left(n_{1} n_{2}\left(\frac{\partial^{2} u}{\partial x_{1}^{2}}-\frac{\partial^{2} u}{\partial x_{2}^{2}}\right)-\left(n_{1}^{2}-n_{2}^{2}\right) \frac{\partial^{2} u}{\partial x_{1} \partial x_{2}}\right) \\
& B_{2}(u)=n_{1}^{2} \frac{\partial^{2} u}{\partial x_{1}^{2}}+n_{2}^{2} \frac{\partial^{2} u}{\partial x_{2}^{2}}+2 n_{1} n_{2} \frac{\partial^{2} u}{\partial x_{1} \partial x_{2}}
\end{aligned}
$$

$\vec{n}=\left(n_{1}, n_{2}\right)$ is the outer normal to the domain, and $\vec{\sigma}=\left(\sigma_{1}, \sigma_{2}\right)$ the tangent vector such that $(\vec{n}, \vec{\sigma})$ forms an orthonormal basis.

In mechanics $B_{1}(u)$ is the transverse force (shear force and twisting moment), $B_{2}(u)$ represents the bending moment and $u$ is the deflection of the thin plate. For more details on this problem we refer the reader to $[12,15]$.

REMARK 1. By using a maximum a posteriori estimator (MAP estimator), we can show that the data fidelity term in (2.2) is adapted to the model $f=u+b$ where $f$ is the observed image, $u$ the image to recover and $b$ a Gaussian noise.

3. Computation of the topological gradient in cases (a) of a perforated domain and (b) of a cracked domain. The following theorem is taken from [13] and adapted to a numerical objective. In [13] we give the topological gradient expression in the cases of perforated and cracked domains. The calculus of the topological gradient relies upon the evaluation of the difference $J_{\epsilon}\left(u_{\epsilon}\right)-J_{0}\left(u_{0}\right)$ when $\epsilon \rightarrow 0$. This evaluation is very technical but (and it is remarkable) only needs the computation of two solutions : $u_{0}$ the solution of the direct problem $\left(\mathcal{P}_{0}\right)$ and $v_{0}$ the solution of the adjoint problem $\left(\mathcal{Q}_{0}\right)$ defined by

$$
\left(\mathcal{P}_{0}\right)\left\{\begin{aligned}
\alpha \Delta^{2} u_{0}+u_{0} & =f \text { in } \Omega \\
B_{1}\left(u_{0}\right) & =B_{2}\left(u_{0}\right)=0 \text { on } \partial \Omega
\end{aligned}\right.
$$

and

$$
\left(\mathcal{Q}_{0}\right)\left\{\begin{aligned}
\alpha \Delta^{2} v_{0}+v_{0} & =2 u_{0}-f \text { in } \Omega \\
B_{1}\left(v_{0}\right) & =B_{2}\left(v_{0}\right)=0 \text { on } \partial \Omega
\end{aligned}\right.
$$

REMARK 2. In the adjoint problem, the source term is $2 u_{0}-f$ and so $v_{0}$ is closed to $f$ if $u_{0}$ is closed to $f$.

THEOREM 3.1.

The topological gradients of the cost function (2.1) associated with the PDE (2.5) in the cases of a perforated domain (denoted by $\mathcal{I}^{b}$ ) and a cracked domain (denoted by $\mathcal{I}^{c}$ ) are:

$$
\mathcal{I}^{b}\left(x_{0}\right)=\frac{\pi}{\alpha}\left(f\left(x_{0}\right)-u_{0}\left(x_{0}\right)\right)\left(v_{0}\left(x_{0}\right)-u_{0}\left(x_{0}\right)\right)-\mathcal{M}\left(u_{0}, v_{0}\right)\left(x_{0}\right)
$$

with

$$
\mathcal{M}(u, v)=\pi \Delta u \Delta v+\frac{2 \pi}{3}\left(\left(\frac{\partial^{2} u}{\partial x_{1}^{2}}-\frac{\partial^{2} u}{\partial x_{2}^{2}}\right)\left(\frac{\partial^{2} v}{\partial x_{1}^{2}}-\frac{\partial^{2} v}{\partial x_{2}^{2}}\right)+4 \frac{\partial^{2} u}{\partial x_{1} \partial x_{2}} \frac{\partial^{2} v}{\partial x_{1} \partial x_{2}}\right)
$$

and

$$
\mathcal{I}^{c}\left(x_{0}, \vec{n}\right)=-\frac{2 \pi}{3} \nabla^{2} u_{0}\left(x_{0}\right)(\vec{n}, \vec{n}) \nabla^{2} v_{0}\left(x_{0}\right)(\vec{n}, \vec{n})
$$


where $\vec{n}$ is the normal to the crack, $u_{0}$ and $v_{0}$ are the solutions of the direct and dual problems $\left(\mathcal{P}_{0}\right)$ and $\left(\mathcal{Q}_{0}\right)$ given by $(3.1)$ and $(3.2)$ respectively. We define the topological indicators at point $x_{0}$ for a perforated and cracked domain as

$$
\begin{gathered}
I_{\text {Bilap }}^{b}\left(x_{0}\right)=\left|\mathcal{I}^{b}\left(x_{0}\right)\right| \\
I_{\text {Bilap }}^{c}\left(x_{0}\right)=\max _{\|\vec{n}\|=1}\left|\mathcal{I}^{c}\left(x_{0}, \vec{n}\right)\right|
\end{gathered}
$$

We can notice that the computation of the topological gradient for the two models (crack and ball) only needs the resolution of two linear problems $\left(\mathcal{P}_{0}\right)$ and $\left(\mathcal{Q}_{0}\right)$. In section 7 we propose two ways to discretize these problems.

4. Adaptation for a more general problem of deblurring. In most imaging applications the optical material, or the motion of the camera or of the target introduce blur on the observed image (see [25]). Generally, spatially invariant blur is modeled as a positive convolution operator $u \mapsto K u$ with $K \mathbb{1} \neq 0$. We assume that the observed image is of the form $f=K u+b$ where $K$ is the blur operator, $u$ the image to recover and $b$ a Gaussian additive noise. Thus, we can take into account the blurring by replacing $u$ by $K u$ into the data fidelity terms of the cost function of the variational problem (2.2). Hence, Euler equations become:

$$
\left(\mathcal{P}_{0}\right)\left\{\begin{aligned}
\alpha \Delta^{2} u_{0}+K^{\star} K u_{0} & =K^{\star} f, \text { in } \Omega \\
B_{1}\left(u_{0}\right) & =B_{2}\left(u_{0}\right)=0, \text { on } \partial \Omega
\end{aligned}\right.
$$

and

$$
\left(\mathcal{Q}_{0}\right)\left\{\begin{aligned}
\alpha \Delta^{2} v_{0}+K^{\star} K v_{0} & =K^{\star}\left(2 K u_{0}-f\right), \text { in } \Omega \\
B_{1}\left(v_{0}\right) & =B_{2}\left(v_{0}\right)=0, \text { on } \partial \Omega .
\end{aligned}\right.
$$

For the well posedness of (4.1) and (4.2) we refer the reader to [3] chapter 3. It is straightforward to see that the topological gradient for the cracked domain is still given by the same expression obtained without blur while for a perforated domain its expression is a little bit changed. By still denoting by $I_{\text {Bilap }}^{b}$ and $I_{\text {Bilap }}^{c}$ the two topological indicators associated to (2.1) and to the problem deduced from (2.2) by replacing $u$ by $K u$, we have (see section 3 for notations and [12] for more details) :

$$
\begin{gathered}
I_{\text {Bilap }}^{b}\left(x_{0}\right)=\left|\mathcal{I}^{b}\left(x_{0}\right)\right| \\
I_{\text {Bilap }}^{c}\left(x_{0}\right)=\max _{\|\vec{n}\|=1}\left|\mathcal{I}^{c}\left(x_{0}, \vec{n}\right)\right|
\end{gathered}
$$

with

$$
\begin{aligned}
& \mathcal{I}^{b}\left(x_{0}\right)=\frac{\pi}{\alpha}\left(K^{\star} f\left(x_{0}\right)-K^{\star} K u_{0}\left(x_{0}\right)\right)\left(v_{0}\left(x_{0}\right)-u_{0}\left(x_{0}\right)\right)-\mathcal{M}\left(u_{0}, v_{0}\right)\left(x_{0}\right) \\
& \mathcal{I}^{c}\left(x_{0}\right)=-\frac{2 \pi}{3} \nabla^{2} u_{0}\left(x_{0}\right)(\vec{n}, \vec{n}) \nabla^{2} v_{0}\left(x_{0}\right)(\vec{n}, \vec{n})
\end{aligned}
$$

and where $u_{0}$ and $v_{0}$ are given respectively by (4.1) and (4.2). The illustration of these two topological indicators is performed in section 8 and 9 for $K=I$ (no blur) and in section 10 for $K \neq I$ (with blur). 
5. Restoration using the topological gradient. In this section we present a restoration algorithm preserving edges and filaments. As said in the introduction, the difference between edges and fine structures can be characterized as follows : an edge is a discontinuity with a jump of the intensity and a filament is a discontinuity without jump. The continuous definition of these structures is clear but for numerical images it is no more true. Generally we consider as fine structure, a structure of maximum 4 or 5 pixels width (point or filament). A structure of width more than 5 pixels can be considered as a volume object delimited by edges. We assume that the observed degraded image $f$ writes as $f=K u+b$ where $u$ is the image to recover, $b$ the Gaussian noise and $K$ the blur operator (generally a convolution such that $K \mathbb{1} \neq 0$, see [3] chapter 3). We still denote by $I_{\text {Bilap }}^{c}$ the topological indicator for a cracked domain (4.3b) where $u_{0}$ and $v_{0}$ are given by (4.1) and (4.2) (section 4), and we introduce the topological gradient $I_{L a p}^{c}$ defined in $[8,14]$ for edge detection :

$$
I_{\text {Lap }}^{c}=\min _{\|\vec{n}\|=1} \mathcal{J}^{c}\left(x_{0}, \vec{n}\right) \text { with } \mathcal{J}^{c}\left(x_{0}, \vec{n}\right)=-\pi \nabla u_{0}\left(x_{0}\right) \cdot \vec{n} \nabla v_{0}\left(x_{0}\right) \cdot \vec{n}
$$

where $u_{0}$ and $v_{0}$ are given by

$$
\left\{\begin{array} { r l } 
{ - \gamma \Delta u _ { 0 } + K ^ { \star } K u _ { 0 } } & { = K ^ { \star } f \text { in } \Omega } \\
{ \partial _ { n } u _ { 0 } } & { = 0 \text { on } \partial \Omega }
\end{array} \text { and } \quad \left\{\begin{array}{rl}
-\gamma \Delta v_{0}+K^{\star} K v_{0} & =K^{\star}\left(2 K u_{0}-f\right) \text { in } \Omega \\
\partial_{n} v_{0} & =0 \text { on } \partial \Omega .
\end{array}\right.\right.
$$

with $\gamma>0$ a parameter to tune. As a by-product, the calculus of the topological gradients for a cracked domain $I_{\text {Bilap }}^{c}(4.3 \mathrm{~b})$ and $I_{\text {Lap }}^{c}$ (5.1) allows to restore images degraded by blur or/and Gaussian noise. We propose a restoration preserving both edges and filaments. Once $I_{\text {Bilap }}^{c}$ and $I_{\text {Lap }}^{c}$ are computed, we define for two fixed thresholds $\delta_{1}, \delta_{2}>0$, the sets $E_{\delta_{1}}^{\text {Lap }}=\left\{x \in \Omega ; I_{\text {Lap }}^{c} \geq \delta_{1}\right\}$ and $E_{\delta_{2}}^{\text {Bilap }}=\left\{x \in \Omega ; I_{\text {Bilap }}^{c} \geq \delta_{2}\right\}$ and the characteristic functions

$$
\chi_{\eta}^{\text {Lap }}(x)=\left\{\begin{array}{l}
\eta \text { if } x \in E_{\delta_{1}}^{\text {Lap }} \\
1 \text { otherwise }
\end{array} \quad \text { and } \quad \chi_{\eta}^{\text {Bilap }}(x)=\left\{\begin{array}{l}
\eta \text { if } x \in E_{\delta_{2}}^{\text {Bilap }} \\
1 \text { otherwise }
\end{array}\right.\right.
$$

where $\eta$ is a small positive parameter. From the computation of $I_{\text {Bilap }}^{c}$ and $I_{\text {Lap }}^{c}$, we also get the normalized direction $\vec{\tau}=\vec{n}^{\perp}$ of the discontinuity. For edges, $\vec{n}$ is given by the associated vector to $I_{\text {Lap }}^{c}(5.1)$ and for filaments it is computed by using $I_{\text {Bilap }}^{c}$ (4.3b). If $\vec{n}=(\cos (\varphi), \sin (\varphi))$ is the normal to the minimum crack performed by either $I_{\text {Bilap }}^{c}$ or $I_{\text {Lap }}^{c}$, we denote $\vec{\tau}=(\sin (\varphi),-\cos (\varphi))$. Then if $\mathrm{f}$ is the degraded observed image, the idea is to find a restored version $u$ of $f$ as the solution of the following anisotropic PDE :

$$
\left\{\begin{aligned}
-\operatorname{div}\left(\beta P_{\eta}^{\varphi}(x) \nabla u\right)+K^{\star} K u & =K^{\star} f \text { on } \Omega \\
\partial_{n} u & =0 \text { on } \Gamma
\end{aligned}\right.
$$

where $P_{\eta}^{\varphi}(x)$ is a tensor constructed from $\varphi(x), \chi_{\eta}^{\text {Bilap }}(x)$ and $\chi_{\eta}^{\text {Lap }}(x) ; K$ is the blur operator and $\beta$ is a parameter to tune. More precisely, we choose $P_{\eta}^{\varphi}(x) \nabla u(x)=$ $(\nabla u . \vec{\tau}) \vec{\tau}+\chi_{\eta}(x)(\nabla u \cdot \vec{n}) \vec{n}$ where $\chi_{\eta}$ denotes either $\chi_{\eta}^{\text {Lap }}$ or $\chi_{\eta}^{\text {Bilap }}$ if we want to preserve respectively edges or filaments. A simple identification shows that $P_{\eta}^{\varphi}(x)$ is the matrix (5.4)

$$
P_{\eta}^{\varphi}(x)=\left(\begin{array}{ll}
\sin ^{2}(\varphi(x))+\chi_{\eta}(x) \cos ^{2}(\varphi(x)) & \sin (\varphi(x)) \cos (\varphi(x))\left(\chi_{\eta}(x)-1\right) \\
\sin (\varphi(x)) \cos (\varphi(x))\left(\chi_{\eta}(x)-1\right) & \cos ^{2}(\varphi(x))+\chi_{\eta}(x) \sin ^{2}(\varphi(x))
\end{array}\right)
$$


The interpretation of this matrix $P_{\eta}^{\varphi}(x)$ is as follows :

- if $\mathrm{x}$ belongs to the background thanks to the definition of $\chi_{\eta}(x), P_{\eta}^{\varphi}(x)$ writes as $P_{\eta}^{\varphi}(x)=\left(\begin{array}{ll}1 & 0 \\ 0 & 1\end{array}\right)$ so $\operatorname{div}\left(P_{\eta}^{\varphi}(x) \nabla u\right)=\Delta u$ and the smoothing is isotropic.

- if $\mathrm{x}$ belongs to an edge (i.e. $x \in E_{\delta_{1}}^{L a p}$ ) and if $\chi_{\eta}(x)=\chi_{\eta}^{\operatorname{Lap}}(x)$, then $\chi_{\eta}(x)$ is close to zero and $P_{\eta}^{\varphi}(x) \nabla u(x) \approx(\nabla u . \vec{\tau}) \vec{\tau}$. The diffusion is in the direction of the edge.

- if $\mathrm{x}$ belongs to a filament (i.e. $x \in E_{\delta_{2}}^{\text {Bilap }}$ ) and if $\chi_{\eta}(x)=\chi_{\eta}^{\text {Bilap }}(x)$, then $\chi_{\eta}(x)$ is close to zero and $P_{\eta}^{\varphi}(x) \nabla u(x) \approx(\nabla u \cdot \vec{\tau}) \vec{\tau}$. The diffusion is in the direction of the filament.

REMARK 3. In the case of $\chi_{\eta}=\chi_{\eta}^{\text {Lap }}$, for a more general framework with different kind of noise and blur, we refer the reader to [14].

REMARK 4. If we want to preserve edges and filaments we can choose $\chi_{\eta}(x)=$ $\min \left(\chi_{\eta}^{\text {Lap }}(x), \chi_{\eta}^{\text {Bilap }}(x)\right)$ (see section 10.2).

As we will see in section 10.2 on numerical examples, the restoration results obtained when applying equation (5.3) are very good both for filaments and edges.

6. Comparison with a classical indicator. In $[31,30,24]$ the authors use for detecting the center of vessels an indicator defined from the Hessian matrix of a regularized version of the image. We denote by $\mathrm{f}$ the observed image, $G_{\sigma}$ the Gaussian function of scale $\sigma$ and $H_{\sigma}=\nabla^{2} f * G_{\sigma}=f * \nabla^{2} G_{\sigma}$. If we set $\lambda_{1}$ and $\lambda_{2}$ the eigenvalues of $H_{\sigma}$ such that $0 \leq\left|\lambda_{1}\right| \leq\left|\lambda_{2}\right|$, then we define the two following point and filament indicators :

$$
\begin{aligned}
& I_{H e s}^{p}=\left|\lambda_{2}\right| \text { (points indicator) } \\
& I_{H e s}^{f}=\left|\lambda_{2}\right|-\left|\lambda_{1}\right| \text { (filaments indicator) }
\end{aligned}
$$

$I_{H e s}^{p}(x)$ is high if $\mathrm{x}$ belongs to both points or filaments. $I_{H e s}^{f}(x)$ is high only if there is a discontinuity in exactly one direction i.e. on a filament. With $I_{H e s}^{f}$, we set $v_{1}$ as the associated vector to the smallest eigenvalue of the normalized Hessian matrix [24]

$$
\widehat{H_{\sigma, \rho}}=G_{\rho} *\left[\frac{f * \nabla^{2} G_{\sigma}}{\left(1+\left|f * \nabla G_{\sigma}\right|^{2}\right)^{1 / 2}}\right]
$$

where $\sigma$ and $\rho$ are called respectively the differentiation and the integration scales. In our experiments we set $\rho=0.5$ and $\sigma$ is tuned with respect to the size of the structures we want to detect. This direction is used in [24] to recover the tangent to the filament (or to the center line of the vessel). It is known that the eigenvectors of $H_{\sigma}$ are very sensitive to noise and numerical experiments show that $v_{1}$ matches better the tangent filament direction than the vector associated to the smallest eigenvalue of $H_{\sigma}$. We will compare this direction with the one given by (4.3b)(see section 8.3).

The scale $\sigma$ in all of the pictures is chosen manually in order to have the best result and depends on the size of the structures we want to detect.

7. Discretization of the PDEs (3.1) and (3.2) and numerical calculus of the topological gradient. In this section we present two methods for computing the solutions $u_{0}$ and $v_{0}$ of problems (3.1) and (3.2). The first method uses the P2Morley finite elements and the second one uses the DCT (Discrete Cosine Transform). On the one hand, the convergence of the first method towards the theoretical solution is well known and we will see that it is the least expensive finite elements method in terms of number of variables. On the other hand, we will numerically show that 
if we cannot prove that the second method converges to the solution of problems (3.1) and (3.2), the results are similar and the computing time is much shorter. In this section we denote by $\|u\|_{m, \Omega}$ the norm $\|u\|_{H^{m}(\Omega)}$ where $H^{m}(\Omega)$ is the Sobolev space : $\left\{u, D^{\alpha} u \in L^{2}(\Omega), 0 \leq|\alpha| \leq m\right\}$ where $\alpha \in \mathbb{N}^{d}$ and $|\alpha|=\sum \alpha_{i}$. All calculus are implemented in Matlab 7.5.0 (for the DCT choice and finite difference scheme) and in Freefem ++ (for the $P_{2}$ Morley choice). The experiments are performed on a computer equipped with a processor Intel Core $1.9 \mathrm{GHz}$.

7.1. Discretization by finite elements. The variational formulation of (3.1) and (3.2) are: find $u_{0} \in H(\Omega)$ such that

$$
a_{0}\left(u_{0}, v\right)=\langle f, v\rangle \quad \forall v \in H^{2}(\Omega)
$$

and find $v_{0} \in H^{2}(\Omega)$ such that

$$
a_{0}\left(u, v_{0}\right)=-\left\langle f-2 u_{0}, u\right\rangle \quad \forall u \in H^{2}(\Omega)
$$

where $\langle u, v\rangle$ denotes the $L^{2}(\Omega)$-scalar product. To discretize these two problems we use the nonconforming quadratic Morley finite element (see [23] and [10] pp 148-151 and $\mathrm{p} 305)$. It is defined by the triplet $\left(K, \mathcal{P}_{K}, \Sigma_{K}\right)$ where

(i) $\mathrm{K}$ is a triangle

(ii) $P_{K}$ is the space of polynomial functions of degree less than or equal to 2 defined on $\mathrm{K}$.

(iii) $\Sigma_{K}$ is the set of the following linear maps

$$
\begin{aligned}
& v \mapsto v\left(a_{i}\right), \text { where } a_{i} \text { for } i \in\{1,2,3\} \text { are the vertices of } \mathrm{K} \\
& v \mapsto \partial_{n} v\left(b_{i}\right), \text { where } b_{i} \text { for } i \in\{1,2,3\} \text { are midpoints of } \mathrm{K} \text { edges }
\end{aligned}
$$

and where $b_{i}$ is on the opposite edge of the vertex $a_{i}$ (see Figure 3).

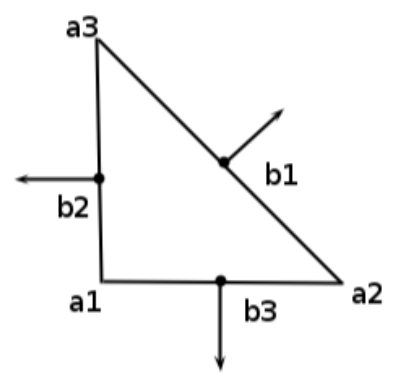

FIG. 3. The quadratic Morley finite element geometry

Let $\left(\mathcal{T}_{h}\right)_{h}$ be a regular triangulation of $\Omega$. For all $h>0$, we denote by $X_{h}$ the Morley finite element space associated with $\left(\mathcal{T}_{h}\right)_{h}$. We introduce the scalar product on $X_{h}$ : $\left\langle u_{h}, v_{h}\right\rangle_{h}=\sum_{T \in \mathcal{T}_{h}} \int_{T} u_{h} v_{h}$ and the discrete norms: $\left\|v_{h}\right\|_{m, h}=\left(\sum_{T \in \mathcal{T}_{h}}\left\|v_{h}\right\|_{m, T}^{2}\right)^{1 / 2}$ for $v_{h} \in X_{h}$. $X_{h}$ :

Let $a_{0}^{h}\left(u_{h}, v_{h}\right), l_{0}^{h}\left(v_{h}\right)$ and $L_{0}^{h}\left(v_{h}\right)$ be the following bilinear and linear forms on 


$$
\begin{aligned}
a_{0}^{h}\left(u_{h}, v_{h}\right) & =\alpha \sum_{i, j \in\{1,2\}}\left\langle\frac{\partial^{2} u_{h}}{\partial x_{i} \partial x_{j}}, \frac{\partial^{2} v_{h}}{\partial x_{i} \partial x_{j}}\right\rangle_{h}+\left\langle u_{h}, v_{h}\right\rangle_{h}, \forall u_{h}, v_{h} \in X_{h}, \\
l_{0}^{h}\left(v_{h}\right) & =\left\langle f, v_{h}\right\rangle_{h}, \forall v_{h} \in X_{h}, \\
L_{0}^{h}\left(v_{h}\right) & =-\left\langle f-2 u_{0, h}, v_{h}\right\rangle_{h}, \forall v_{h} \in X_{h} .
\end{aligned}
$$

We denote by $\left(\mathcal{P}_{0}^{h}\right)$ and $\left(\mathcal{Q}_{0}^{h}\right)$ the two following discrete problems approximating (7.1) and (7.2):

$$
\text { find } u_{0}^{h} \in X_{h} \text { such that: } \quad a_{0}^{h}\left(u_{0}^{h}, v_{h}\right)=l_{0}^{h}\left(v_{h}\right), \forall v_{h} \in X_{h} \quad\left(\mathcal{P}_{0}^{h}\right)
$$

$$
\text { find } v_{0}^{h} \in X_{h} \text { such that: } \quad a_{0}^{h}\left(u_{h}, v_{0}^{h}\right)=L_{0}^{h}\left(u_{h}\right), \forall u_{h} \in X_{h}
$$

We have the following convergence result (see [23]):

THEOREM 7.1. Let $u_{0}$ and $v_{0}$ be the solutions of (7.1) and (7.2) respectively, let $u_{0}^{h}$ and $v_{0}^{h}$ be the solutions of (7.3) and (7.4) respectively, we have the following estimations:

$$
\begin{aligned}
\left\|u_{0}-u_{0}^{h}\right\|_{2, h} & \leq C h\left(\left|u_{0}\right|_{3, \Omega}+h\|f\|_{0, \Omega}\right) \\
\left\|v_{0}-v_{0}^{h}\right\|_{2, h} & \leq C h\left(\left|u_{0}\right|_{3, \Omega}+\left|v_{0}\right|_{3, \Omega}+h\|f\|_{0, \Omega}+h\left\|u_{0}\right\|_{0, \Omega}\right)
\end{aligned}
$$

where $C$ is a constant independent of $h$.

Proof. For the first inequality see [23]. For the second one we split $v_{0}^{h}$ in $v_{0}^{h}=$ $p_{0}^{h}+q_{0}^{h}$, where $p_{0}^{h}$ and $q_{0}^{h}$ are solutions of

$$
\begin{aligned}
& a_{0}^{h}\left(u_{h}, p_{0}^{h}\right)=-\left\langle f-2 u_{0}, u_{h}\right\rangle, \quad \forall u_{h} \in X_{h}, \\
& a_{0}^{h}\left(u_{h}, q_{0}^{h}\right)=-2\left\langle u_{0}-u_{0}^{h}, u_{h}\right\rangle, \quad \forall u_{h} \in X_{h} .
\end{aligned}
$$

Thanks to [23], it is straightforward to see that

$$
\left\|p_{0}^{h}-v_{0}\right\|_{2, h} \leq C h\left(\left|v_{0}\right|_{3, \Omega}+h\left\|f-2 u_{0}\right\|_{0, \Omega}\right) .
$$

Since $a_{0}^{h}$ is coercive on $X_{h}$ and by setting $u_{h}=q_{0}^{h}$, we get

$$
\left\|q_{0}^{h}\right\|_{2, h} \leq C\left\|u_{0}-u_{0}^{h}\right\|_{2, h} .
$$

By using the fact that $\left\|v_{0}^{h}-v_{0}\right\|_{2, h} \leq\left\|p_{0}^{h}-v_{0}\right\|_{2, h}+\left\|q_{0}^{h}\right\|_{2, h}$ and the first estimation, we get the result.

This finite element has 6 degrees of freedom which corresponds to the number of $P_{2}$ elements in $2 \mathrm{D}$. There are $\mathcal{O}(2 N)$ elements if $\mathrm{N}$ denotes the number of vertices. The matrix of the linear system is sparse and so the solving cost of the system is $\mathcal{O}(N)$ if we use for example a $L U$ method with band matrices.

REMARK 5. It is known (see [10], pp 148-151 and p 305) that this non-conform finite element has the advantage of being less expansive than a conform one (the $P_{5}$ Argyris finite element, which has 21 degrees of freedom). 
7.2. Discretisation by the DCT. As said in the introduction of this section, the DCT does not solve exactly the real problems (7.1) and (7.2) (Neumann conditions are a priori not fulfilled), but looking at the results (see section 8 and 9) and the computation time (see Figure 4), this method presents the serious advantage of being very fast. The theoretical computation time consists in $\mathcal{O}(N \log (N))$ operations for the DCT if $N$ denotes the number of pixels (or voxels) in the image while for the quadratic Morley finite element the cost is $\mathcal{O}(N)$. However, Figure 4 shows that the DCT is much faster than the P2-Morley finite element method for $N \leq 10^{9}$.

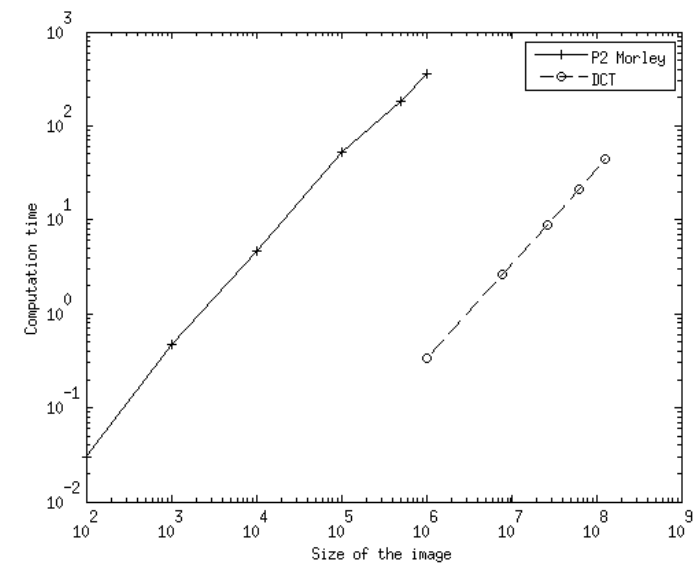

FIG. 4. Variation of the $u_{0}$ and $v_{0}$ computational time for $u_{0}$ and $v_{0}$ according to the size of the image using the logarithmic scale for both DCT and P2-Morley methods.

Considering that the image is a periodic signal, we choose to discretize (3.1) and (3.2) with a DCT2 because of the symmetry properties guaranted by this algorithm. Moreover it is the most classical algorithm used in image processing. We can see the image as a half part of a periodic and symmetric signal. To fix ideas, in 1D if the input image is the sequence of $\mathrm{N}$ points $\left[x_{0} x_{1} \ldots x_{N-1}\right]$, the associated signal is the sequence of $2 N-2$ points $\left[x_{0} x_{1} \ldots x_{N-1} x_{N-2} \ldots x_{1}\right]$. In $1 \mathrm{D}$ the trigonometric function associated with a vector of DCT2 coefficients is

$$
u^{N}(x)=\sum_{k=0}^{N-1} \widehat{y_{k}^{N}} \cos \left(\frac{2 \pi\left(k+\frac{1}{2}\right) x}{T}\right)
$$

where $\widehat{y^{N}} \in \mathbb{R}^{N}$ is the vector such that $\widehat{y^{N}}=D C T 2\left(y^{N}\right), y^{N}=\left(u^{N}(l T / 2 N)\right)_{l}$ is the vector composed by the values of the interpolating function $u$.

From (7.6), it is straightforward to see that the derivatives of $u(x)$ evaluated at points $x_{l}=\frac{l T}{2 N}$ are given by the Fourier coefficients of the function. To fix ideas let us write the fourth derivative with respect to $\mathrm{x}$ of the function $\mathrm{u}$ given by (7.6) :

$$
\frac{d u^{N}}{d x^{4}}(x)=\sum_{k=0}^{N-1} \widehat{y_{k}^{N}}\left(\frac{2 \pi\left(k+\frac{1}{2}\right)}{T}\right)^{4} \cos \left(\frac{2 \pi\left(k+\frac{1}{2}\right) x}{T}\right)
$$

Hence by setting $x=\frac{l T}{N}$, we get $\frac{d u^{N}}{d x^{4}}\left(\frac{l T}{N}\right)$ as a function of $\widehat{y_{k}^{N}}$. Let us do the same thing in 2D. By considering the image as a signal $2\left(N_{x}-1\right)$-periodic with 
respect to $\mathrm{x}$, and 2( $\left.N_{y}-1\right)$-periodic with respect to $\mathrm{y}$, we define the frequency domain by $\left\{\left(\frac{\pi\left(k_{x}+\frac{1}{2}\right)}{\left(N_{x}-1\right)}, \frac{\pi\left(k_{y}+\frac{1}{2}\right)}{\left(N_{y}-1\right)}\right),\left(k_{x}, k_{y}\right) \in E_{x} \times E_{y}\right\}$ with $E_{x}=\left\{0, \ldots, N_{x}-1\right\}$ and $E_{y}=$ $\left\{0, \ldots, N_{y}-1\right\}$. We denote by $\Lambda_{x}$ and $\Lambda_{y}$ the $2\left(N_{y}-1\right) \times 2\left(N_{x}-1\right)$ mesh grids associated with this discrete space. The vector of Fourier coefficients associated to a discrete signal $x \in \mathbb{R}^{N}$ is denoted by $\mathrm{X}$.

Algorithm 1 Computation of the solutions (3.1) and (3.2)

Given an image $f_{i j}$ defined for $\left.(i, j) \in \llbracket 0, N_{y}-1\right) \rrbracket \times \llbracket 0, N_{x}-1 \rrbracket$

1. Use a DCT2 to compute $F_{k l}$ for $(k, l) \in \llbracket 0, N_{y}-1 \rrbracket \times \llbracket 0, N_{x}-1 \rrbracket$.

2. Given $\Lambda$ the meshgrid associated to the frequencies domain described in section 7.2 compute :

$$
\begin{gathered}
\Delta_{k l}^{2}=\Lambda_{x k l}^{4}+2 \Lambda_{x k l}^{2} \Lambda_{y k l}^{2}+\Lambda_{y k l}^{4} \\
U_{k l}=\frac{F_{k l}}{1+\alpha \Delta_{k l}^{2}} \text { and } V_{k l}=\frac{2 U_{k l}-F_{k l}}{1+\alpha \Delta_{k l}^{2}}
\end{gathered}
$$

3. Use an inverse DCT2 to compute $u_{i j}$ and $v_{i j}$ for $(i, j) \in \llbracket 0, N_{y}-1 \rrbracket \times \llbracket 0, N_{x}-1 \rrbracket$.

7.3. Algorithm for the computation of the topological indicators. One of the advantages of the topological gradient method is the simplicity of the final algorithm. To compute (4.3b), we rewrite the topological gradient for a crack of normal $\vec{n}=(\cos (\varphi), \sin (\varphi))$ as

$\mathcal{I}_{c}\left(x_{0}, \varphi\right)=-\frac{2 \pi}{3} P\left(\partial_{x x} u_{0}^{N}\left(x_{0}\right), \partial_{y y} u_{0}^{N}\left(x_{0}\right), \partial_{x y} u_{0}^{N}\left(x_{0}\right), \varphi\right) \times P\left(\partial_{x x} v_{0}^{N}\left(x_{0}\right), \partial_{y y} v_{0}^{N}\left(x_{0}\right), \partial_{x y} v_{0}^{N}\left(x_{0}\right), \varphi\right)$

where $P: \mathbb{R}^{4} \longrightarrow \mathbb{R}$ is the following $\pi$-periodical function with respect to the last variable $\varphi$

$$
P(a, b, c, \varphi)=\frac{1}{2}(a+b)+\frac{1}{2}(a-b) \cos (2 \varphi)+c \sin (2 \varphi) .
$$

Then the computation of (4.3b) comes from the minimization of $\mathcal{I}_{c}\left(x_{0}, \varphi\right)$ with respect to $\varphi \in[0, \pi]$. Derivatives of $u_{0}^{N}$ and $v_{0}^{N}$ are computed by a convolution with derivative filters which is faster than using of matrices/vector product. It is easier to compute (4.3a) than (4.3b) (for $K=I$ ) since the formula is an explicit function of the discrete solutions $u_{0}^{N}$ and $v_{0}^{N}$. Finally we sum up the computation of the topological indicators $I_{\text {Bilap }}^{b}$ (4.3a) and $I_{\text {Bilap }}^{c}$ (4.3b) for $K=I$ in Algorithm 2:

Algorithm 2 Computation of $I_{\text {Bilap }}^{b}(4.3 \mathrm{a})$ and $I_{\text {Bilap }}^{c}(4.3 \mathrm{~b})(K=I)$

1. Computation of $u_{0}^{N}$ and $v_{0}^{N}$ the numerical approximation of the solutions of (3.1) and (3.2) by using finite elements or DCT.

2. Computation of the topological indicator by using (4.3a) or (4.3b) for $K=I$. 
8. Application to filament detection on images possibly noisy. In this section we give some numerical results on synthetic images and real images. In all of this section we assume that there is no blur $(K=I)$. In this section we compare the four indicators $I_{\text {Bilap }}^{b}$ (4.3a), $I_{\text {Bilap }}^{c}$ (4.3b), $I_{\text {Lap }}^{c}$ (5.1) and $I_{\text {Hes }}^{f}(6.1)$ on images containing filaments, road or vessel-like structures. We will display these different indicators on edges and on filaments in order to illustrate Lemma 2.1. Then, we study the robustness of $I_{\text {Bilap }}^{b}, I_{\text {Bilap }}^{c}$ and $I_{\text {Hes }}^{f}$ with respect to noise.

To visualize positive indicators on gray level images, we display their opposite value rescaled in $[0,255]$ in order to have the detected structures in black and the rest in white.

8.1. Structures detected by the topological indicators. In this section we compare the fourth indicators on a simple synthetic image and on a real one containing edges and filaments. Figure 5 compares, in a simple case, the bilaplacian and the Laplacian indicators with the indicator $I_{\text {Hes }}^{f}$ on an edge and on a filament. $I_{\text {Bilap }}^{b}, I_{\text {Bilap }}^{c}$ and $I_{\text {Hes }}^{f}$ are more sensitive to filaments than to edges, contrary to $I_{\text {Lap }}^{c}$. This simple case numerically justifies the second order derivatives in the cost function (2.1) and it is also a numerical validation of Lemma 2.1. In a similar way, Figure 6 shows a real initial image with the three indicators. $I_{\text {Bilap }}^{b}$ and $I_{\text {Bilap }}^{c}$ mainly detect the center of the road network. $I_{\text {Hes }}$ is also higher on roads than on edges but a lot of noise and texture are detected. $I_{\text {Lap }}^{c}$ is high on edges and on the boundaries of the road.

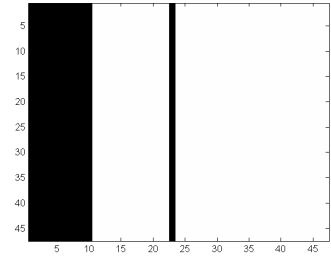

(a) Initial Image

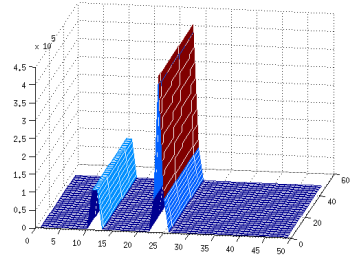

(b) $I_{\text {Bilap }}^{c}\left(\alpha=10^{-3}\right)$

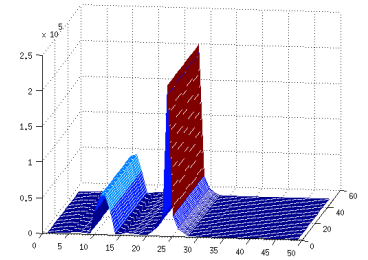

(c) $I_{\text {Bilap }}^{b}(\alpha=0.1)$

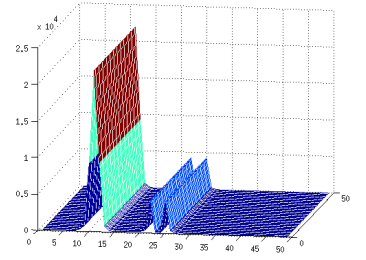

(d) $I_{\text {Lap }}^{c}(\alpha=0.1)$

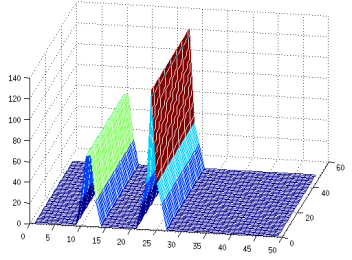

(e) $I_{H e s}^{f}(\sigma=3 / 4)$

FIG. 5. Visualization of $I_{\text {Bilap }}^{b}(4.3 \mathrm{a}), I_{\text {Bilap }}^{c}(4.3 \mathrm{~b}), I_{\text {Lap }}^{c}(5.1)$ and $I_{\text {Hes }}^{f}(6.1)$ on an edge and a filament on a synthetic image 


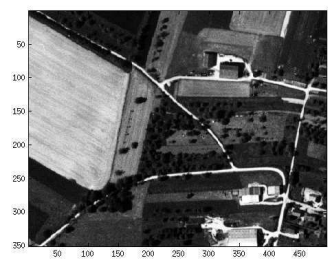

(a) Initial Image

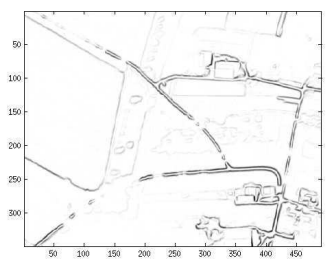

(b) $I_{\text {Lap }}^{c}(\alpha=0.1)$

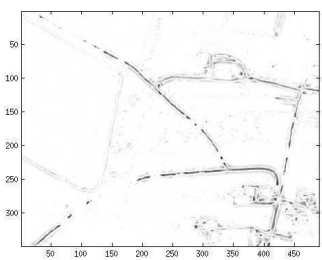

(c) $I_{\text {Bilap }}^{b}(\alpha=1)$

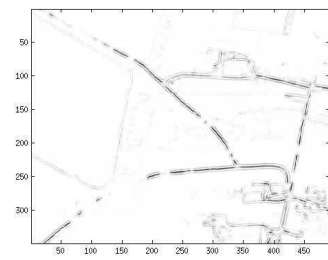

(d) $I_{\text {Bilap }}^{c}\left(\alpha=10^{-2}\right)$

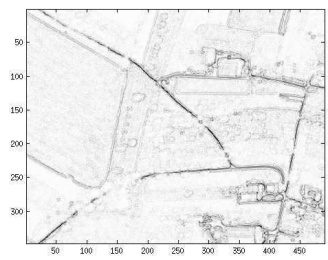

(e) $I_{\text {Hes }}^{f}(\sigma=5 / 4)$

FIG. 6. Comparison of $I_{\text {Bilap }}^{b}$ (4.3a), $I_{\text {Bilap }}^{c}$ (4.3b), $I_{\text {Lap }}^{c}$ (5.1) and $I_{\text {Hes }}^{f}$ (6.1) on a real image

These results show that our model is adapted to the filament detection. In the next subsection we study and compare the robustness of $I_{\text {Bilap }}^{b}, I_{\text {Bilap }}^{c}$ and $I_{\text {Hes }}^{f}$ with respect to noise (of type Gaussian).

8.2. Robustness of the topological gradient with respect to Gaussian noise and comparisons. An important quality of an indicator is its robustness with respect to noise. In this section, we compare the robustness with respect to noise of $I_{\text {Bilap }}^{b}, I_{\text {Bilap }}^{c}$ and $I_{\text {Hes }}^{f}$. Figure 7 displays a profile of a noisy filament, the direct solution (3.1), the adjoint solution (3.2) and $I_{\text {Bilap }}^{c}(4.3 \mathrm{~b})$. We see that some aliasing appears for the direct and adjoint solutions. This is due to the truncation in the Fourier serie. Figure 8 displays these three indicators for a synthetic image with three different PSNR (peak signal-to-noise ratio). We recall that in all experiments only Gaussian noise is used. This study shows that $I_{\text {Bilap }}^{c}$ and $I_{\text {Bilap }}^{b}$ are more robust with respect to noise than the indicator $I_{\text {Hes }}^{f}$. Next $I_{\text {Bilap }}^{c}$ is more robust than $I_{\text {Bilap }}^{b}$. The tuning of $\alpha$ depends on the size of the structures to detect and to the level of noise. The more $\alpha$ is increased the more the topological gradient is spread and smooth. 


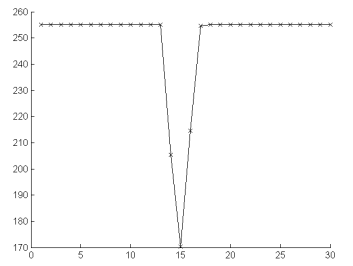

(a) Initial image

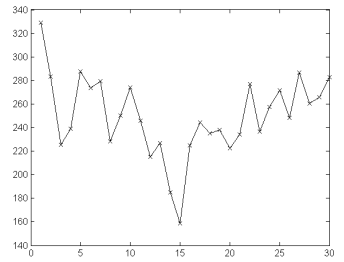

(b) Noisy image

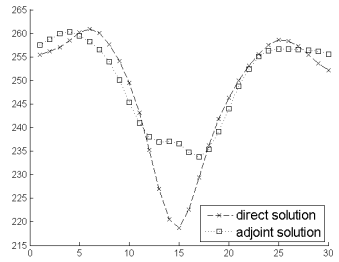

(c) (3.1) and (3.2)

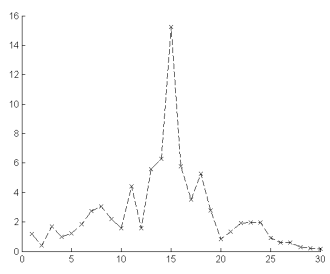

(d) $(4.3 \mathrm{~b})$

FIG. 7. A transverse cut displaying (a) the initial image, (b) its noisy version (PSNR=26dB), (c) the direct and adjoint solutions and (d) $I_{\text {Bilap }}^{c}$ for $\alpha=0.8$

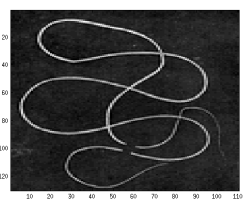

(a) Initial Image (no noisy)

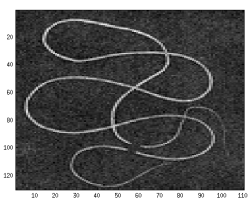

(e) Initial Image $(\mathrm{PSNR}=26 \mathrm{~dB})$

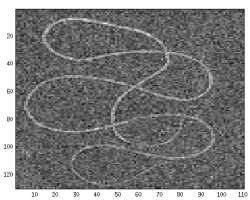

(i) $\quad$ Initial
$(\mathrm{PSNR}=14 \mathrm{~dB})$

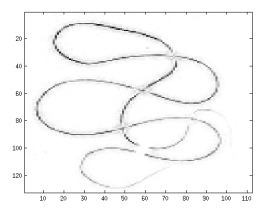

(b) $I_{\text {Bilap }}^{b}(\alpha=0.1)$

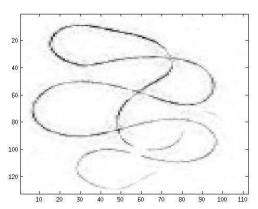

(f) $I_{\text {Bilap }}^{b}(\alpha=0.6)$

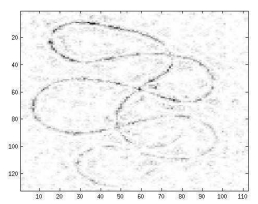

(j) $I_{\text {Bilap }}^{b}(\alpha=1)$

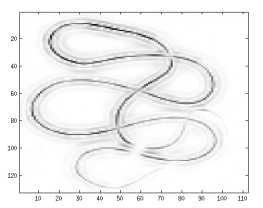

(c) $I_{\text {Bilap }}^{c}(\alpha=0.5)$

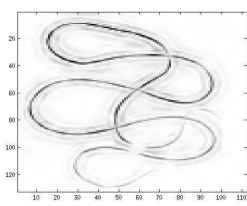

(g) $I_{\text {Bilap }}^{c}(\alpha=1)$

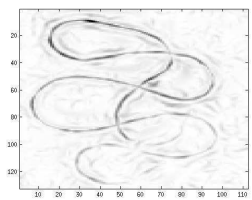

(k) $I_{\text {Bilap }}^{c}(\alpha=3)$

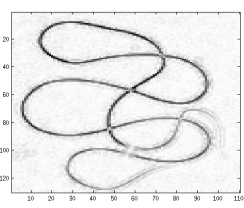

(d) $I_{H e s}^{f}(\sigma=5 / 4)$

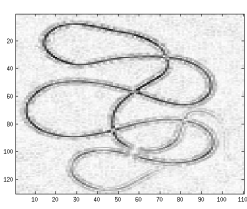

(h) $I_{H e s}^{f}(\sigma=5 / 4)$

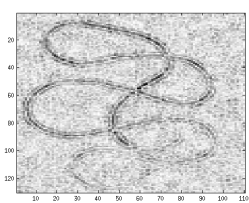

(l) $I_{H e s}^{f}(\sigma=5 / 4)$

FIG. 8. Study of the robustness with respect to noise of $I_{\text {Bilap }}^{b}(4.3 \mathrm{a}), I_{\text {Bilap }}^{c}(4.3 \mathrm{~b})$ and $I_{\text {Hes }}^{f}$ (6.1) on a synthetic image. Each row matches a level of noise and each column is associated to an indicator. 
8.3. Additional information given by the computation of $I_{\text {Bilap }}^{c}(4.3 \mathrm{~b})$. Two important data for filament detection are its location and its direction. In this section we present another advantage of the crack model : its computing (4.3b) leads to finding an optimal crack direction that matches the filament direction. We recall that the computation of $I_{\text {Hes }}^{f}$ gives also an approximation of the direction but it is sensitive to noise. In [24] the authors compute the associated vector to the smallest eigenvalue of the normalized Hessian matrix recalled in section 6 . We still use the same notations in section 6 , and we denoted by $v_{1}$ the vector obtained with this method. In this subsection we compare this vectors field with the orthogonal direction of the vector maximizing (4.3b).

Figure 9 displays the vectors field performed by (4.3b) and the vectors field $v_{1}$. We can see that the vectors given by (4.3b) match better the filament tangent vectors and seems more robust with respect to noise than the vectors $v_{1}$. Moreover the computation of $v_{1}$ needs to tune two parameters $\rho$ and $\sigma$. Besides this method is efficient only for images degraded by Gaussian noise while the topological gradient method can be adapted to the more general problem of deblurring by changing the data fidelity term in (2.1) (see sections 4 and 10).

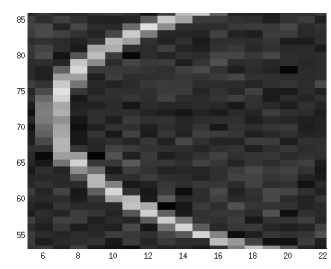

(a) Initial noisy $(\mathrm{PSNR}=26 \mathrm{~dB})$

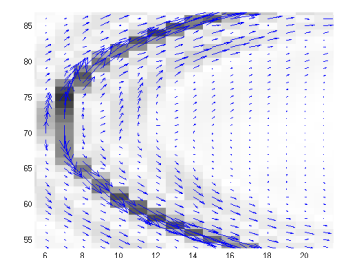

image (b) Vectors field associated with $I_{\text {Bilap }}^{c}(4.3 \mathrm{~b})$

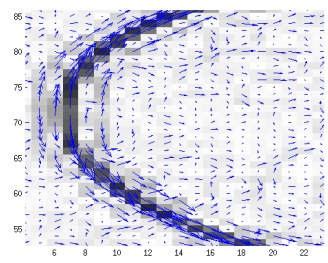

(c) Vectors field $v_{1}[24]$

FIG. 9. The tangent vectors field given by (4.3b) compared to the vectors field $v_{1}$ [24]. For (b) the length of the vectors is proportional to $I_{\text {Bilap }}^{c}$ and for (c) it is proportional to $I_{H e s}^{f}(6.1)$.

9. Applications to point detection on images possibly noisy. In this section we focus on point detection. In all of this section we consider that there is no blur i.e. $K=I$. We assume here that points are circular structures of few pixels $(2$ or 3 pixels). We focus on structures that do not have a privileged direction (isotropic structures). In this section we will compare the indicators $I_{\text {Lap }}^{c}(5.1), I_{\text {Bilap }}^{b}$ (4.3a), $I_{\text {Bilap }}^{c}(4.3 \mathrm{~b})$ and $I_{\text {Hes }}^{p}(6.1)$. We first compare these fourth indicators on a non noisy and simple image containing a small and a big circular structures. Then we compare their robustness with respect to Gaussian noise. We recall that to visualize positive indicators on gray level images, we display their opposite value rescaled in $[0,255]$.

9.1. Structures detected by the topological gradient. Figure 10 illustrates Lemma 2.1. It displays $I_{\text {Lap }}^{c}, I_{\text {Bilap }}^{c}, I_{\text {Bilap }}^{b}$ and $I_{\text {Hes }}^{p}$ on an image containing a big and a small isotropic structure. As expected we see that $I_{\text {Lap }}^{c}$ is inefficient to detect small structures : only the edges are detected and not the center. For respectively different values of $\alpha, I_{\text {Bilap }}^{b}$ and $I_{\text {Bilap }}^{c}$ give similar results in the case of a non noisy image. Indeed we observe that $I_{\text {Bilap }}^{b}$ for $\alpha=0.1$ and $I_{\text {Bilap }}^{c}$ for $\alpha=10^{-3}$ are more sensitive to small objects (about 2 or 3 pixels, e.g. small cells ) than to big ones, while $I_{\text {Lap }}^{c}$ is more sensitive to big objects (about 10 pixels) than to small ones. $I_{H e s}^{p}$ for $\sigma=5 / 4$ 
detects at the same level the center of the small structure and the edges of the big one.

Figure 10 and Figure 11 show that by increasing $\alpha$, the size of structures detected by $I_{\text {Bilap }}^{b}$ and $I_{\text {Bilap }}^{c}$ increases. Hence we deduce that $\alpha$ must be tuned with respect to the size of the structures we want to detect. $I_{\text {Hes }}^{p}$ is singular on both small and big structures but the contrast between the background and the detected structures is lower than for the topological indicators. All of this shows that the choice of the topological model (2.1)-(2.2) is adapted to the point detection.

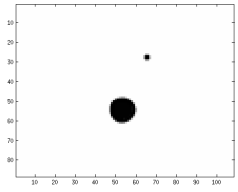

(a) Initial Image

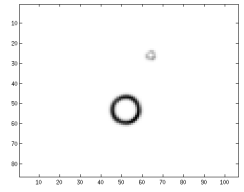

(b) $I_{\text {Lap }}^{c}(\alpha=0.1)$

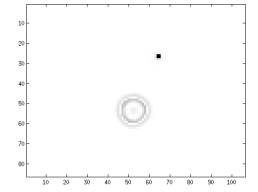

(c) $I_{\text {Bilap }}^{b}(\alpha=0.1)$

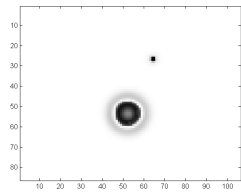

(d) $I_{\text {Bilap }}^{b}(\alpha=3)$

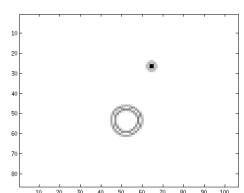

(e) $I_{\text {Bilap }}^{c}\left(\alpha=10^{-3}\right)$

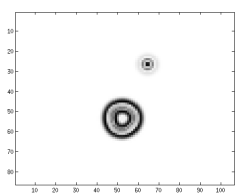

(f) $I_{\text {Bilap }}^{c}(\alpha=0.1)$

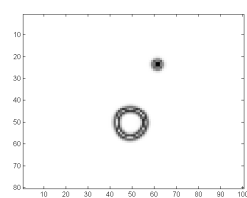

(g) $I_{H e s}^{p}(\sigma=5 / 4)$

FIG. 10. Comparison of $I_{\text {Lap }}^{c}$ (5.1), $I_{\text {Bilap }}^{b}$ (4.3a), $I_{\text {Bilap }}^{c}(4.3 \mathrm{~b})$ and $I_{\text {hes }}^{p}$ (6.1) on a simple synthetic image without noise

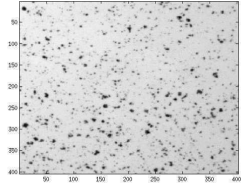

(a) Initial Image

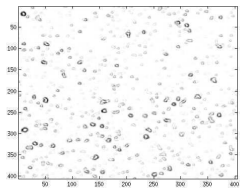

(b) $I_{\text {Lap }}^{c}(\alpha=0.1)$

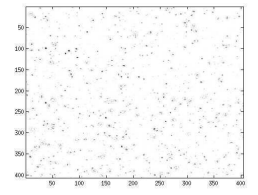

(c) $I_{\text {Bilap }}^{b}(\alpha=0.1)$

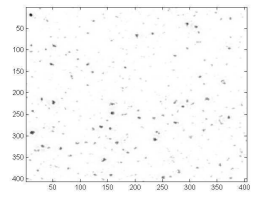

(d) $I_{\text {Bilap }}^{b}(\alpha=3)$

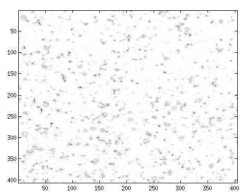

(e) $I_{\text {Bilap }}^{c}\left(\alpha=10^{-3}\right)$

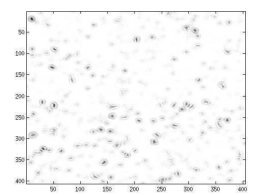

(f) $I_{\text {Bilap }}^{c}(\alpha=0.1)$

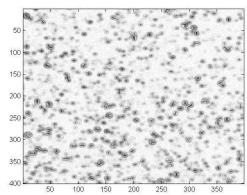

(g) $I_{H e s}(\sigma=5 / 4)$

FIG. 11. Comparison of $I_{\text {Lap }}^{c}(5.1), I_{\text {Bilap }}^{b}(4.3 \mathrm{a}), I_{\text {Bilap }}^{c}(4.3 \mathrm{~b})$ and $I_{\text {hes }}^{p}$ (6.1) on a real image containing cells of different sizes

9.2. Robustness of the topological gradient with respect to Gaussian noise and comparisons. In the previous section we have seen that the point detection needs second order derivatives in (2.1). Now we will compare the robustness of $I_{\text {Bilap }}^{b}$ (4.3a), $I_{\text {Bilap }}^{c}(4.3 \mathrm{~b})$ and $I_{\text {Hes }}^{p}(6.1)$ with respect to Gaussian noise. Figure 
12 shows the indicators $I_{\text {Bilap }}^{b}, I_{\text {Bilap }}^{c}$ and $I_{\text {Hes }}^{p}$ for an image containing two circular objects of different sizes with different levels of noise. Figure 13 shows similar results but for a real non noisy image that we have noised by an additive Gaussian noise. While the results in the case of a non noisy image are similar for $I_{\text {Bilap }}^{b}$ and $I_{\text {Bilap }}^{c}$, it is no more true when we add noise. Indeed if we compare $I_{\text {Bilap }}^{b}$ with $I_{\text {Bilap }}^{c}$, we remark that the fact of increasing $\alpha$ destroys more small structures for $I_{\text {bilap }}^{c}$ than for $I_{\text {Bilap }}^{b}$. We tune the value of the parameter $\alpha$ with the level of noise. When the noise level is high, we increase the value of the parameter $\alpha$ in order to give more importance to the regularization term in (2.2). The tuning of this parameter is very easy with respect to noise. The indicator $I_{H e s}^{p}$ is more sensitive to noise than the topological indicators. According to Figure 12 and Figure 13 the indicator $I_{\text {Bilap }}^{b}$ is the most adapted indicator to detect small isotropic structures. It is quite robust with respect to noise and small structures are detected without a change of their form. $I_{\text {Bilap }}^{c}$ seems to lengthen big structures in one direction while they have not privileged directions. Moreover we must notice that it is easier and faster to compute $I_{\text {Bilap }}^{b}$ (4.3a) than $I_{\text {Bilap }}^{c}$ which is performed by minimizing an expression over the normal $\vec{n}$ of the crack.

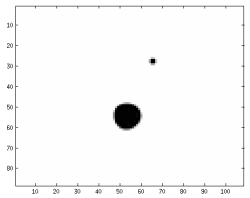

(a) Initial Image, (no noisy)

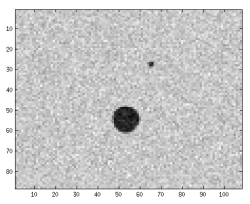

(e) Initial $(\mathrm{PSNR}=20 \mathrm{~dB})$

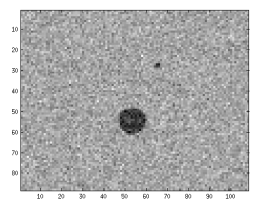

(i) Initial Image $(\mathrm{PSNR}=14 \mathrm{~dB})$

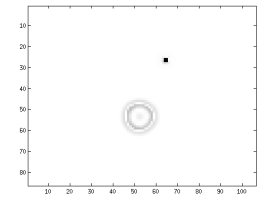

(b) $I_{\text {Bilap }}^{b}(\alpha=0.1)$

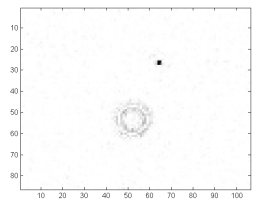

(f) $I_{\text {Bilap }}^{b}(\alpha=0.6)$

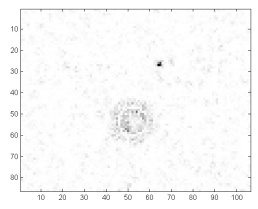

(j) $I_{\text {Bilap }}^{b}(\alpha=1)$

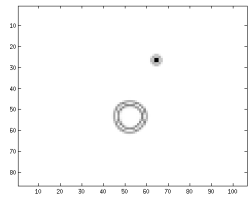

(c) $I_{\text {Bilap }}^{c}\left(\alpha=10^{-3}\right)$

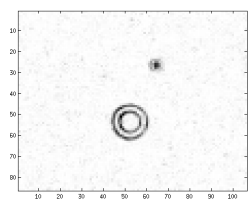

(g) $I_{\text {Bilap }}^{c}\left(\alpha=10^{-2}\right)$

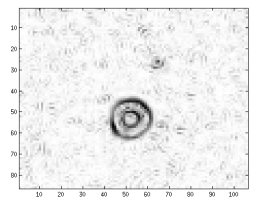

(k) $I_{\text {Bilap }}^{c}\left(\alpha=10^{-1}\right)$

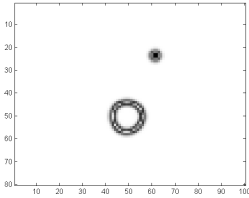

(d) $I_{H e s}^{f}(\sigma=5 / 4)$

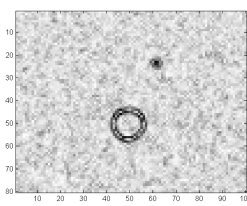

(h) $I_{H e s}^{f}(\sigma=5 / 4)$

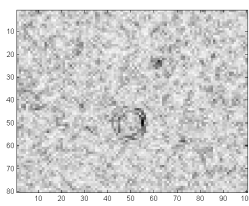

(l) $I_{\text {Hes }}^{f}(\sigma=5 / 4)$

FIG. 12. Study of the robustness with respect to noise of $I_{\text {Bilap }}^{b}(4.3 \mathrm{a}), I_{\text {Bilap }}^{c}(4.3 \mathrm{~b})$ and $I_{\text {hes }}^{p}$ (6.1) on a synthetic image. Each row matches a level of noise and each column is associated to an indicator 


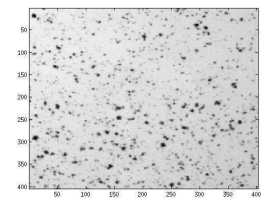

(a) Initial Image (no noisy)

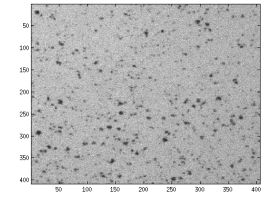

(e) Initial $(\mathrm{PSNR}=20 \mathrm{~dB})$

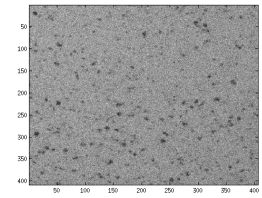

(i) Initial Image $(\mathrm{PSNR}=14 \mathrm{~dB})$

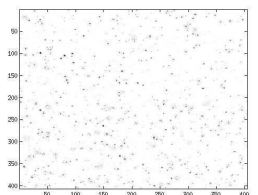

(b) $I_{\text {Bilap }}^{b}(\alpha=0.1)$

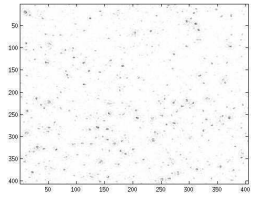

(f) $I_{\text {Bilap }}^{b}(\alpha=0.6)$

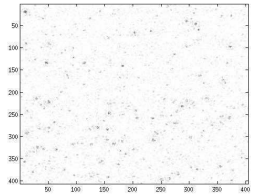

(j) $I_{\text {Bilap }}^{b}(\alpha=1)$

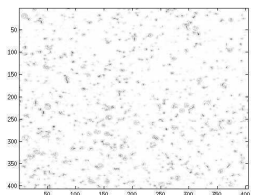

(c) $I_{\text {Bilap }}^{c}\left(\alpha=10^{-3}\right)$

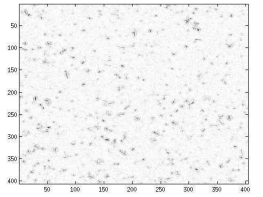

(g) $I_{\text {Bilap }}^{c}\left(\alpha=10^{-2}\right)$

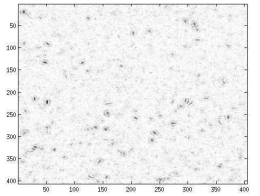

(k) $I_{\text {Bilap }}^{c}\left(\alpha=10^{-1}\right)$

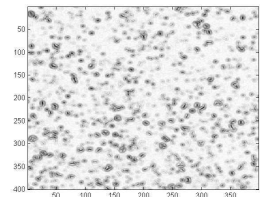

(d) $I_{H e s}(\sigma=5 / 4)$

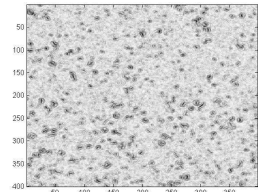

(h) $I_{H e s}(\sigma=5 / 4)$

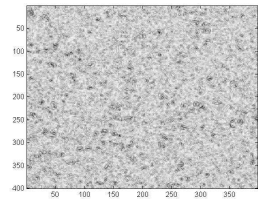

(l) $I_{\text {Hes }}(\sigma=5 / 4)$

FIG. 13. Study of the robustness with respect to noise of $I_{\text {Bilap }}^{b}(4.3 \mathrm{a}), I_{\text {Bilap }}^{c}(4.3 \mathrm{~b})$ and $I_{\text {hes }}^{p}$ (6.1) on a real non nosiy image and on its noisy versions. Each row matches a level of noise and each column is associated to an indicator

10. Application to blurred images : segmentation and restoration. In this section we present how to discretize (4.1) and (4.2) (for $K \neq I$ ) and we present numerical results for both points detection and filaments detection for images contamined by blur and noise, and we illustrate the restoration model (5.3).

10.1. Discretisation by FFT. One property not full filled by the DCT2 is that the DCT2 of a convolution product of two functions is not the DCT2 product of each function. However we know that the property is guaranted by the DCT1. A DCT1 of $\mathrm{N}$ points is equivalent to a DFT of $2 \mathrm{~N}-2$ points. To fix ideas in $1 \mathrm{D}$ for $N=5$, the DCT1 of the row vector $\left[x_{0}, x_{1}, x_{2}, x_{3}, x_{4}\right]$ is equivalent to an FFT of the row vector $\left[x_{0}, x_{1}, x_{2}, x_{3}, x_{4}, x_{3}, x_{2}, x_{1}\right]$. Hence we extend the initial $N_{y} \times N_{x}$ image to an $2(N y-1) \times 2(N x-1)$ image. We consider that the image is a signal $2(N x-1)$-periodic with respect to $\mathrm{x}$ and $2(N y-1)$-periodic with respect to $\mathrm{y}$. As said in section 7.2 the advantage of this extension is that the trigonometric interpolation of the signal is regular across the boundary of the extended image and symmetric by construction. It is the most classical extension in image processing and it allows to avoid "edge effect". In the sequel we denote by X the Fourrier coefficient vector of the vector $\mathrm{x}$. To avoid aliasing introduced by the bilaplacian operator we take the following space of frequencies (see [17]): $\left\{\left(\frac{\pi k_{x}}{\left(N_{x}-1\right)}, \frac{\pi k_{y}}{\left(N_{y}-1\right)}\right),\left(k_{x}, k_{y}\right) \in E_{x} \times E_{y}\right\}$ with $E_{x}=\left\{0, \ldots, N_{x}-1,-\left(N_{x}-2\right), \ldots,-1\right\}$ and $E_{y}=\left\{0, \ldots, N_{y}-1,-\left(N_{y}-2\right), \ldots,-1\right\}$. We denote by $\Lambda_{x}$ and $\Lambda_{y}$ the $2\left(N_{y}-1\right) \times 2\left(N_{x}-1\right)$ mesh grids associated with this 
discrete space. To compute the DFT of the convolution kernel we can either

- extend the kernel $k_{i j}$ defined on $\llbracket 0,2 n_{x} \rrbracket \times \llbracket 0,2 n_{y} \rrbracket$ centered at $\left(n_{x}, n_{y}\right)$ in a kernel defined on $\llbracket 0,2\left(N_{x}-1\right)-1 \rrbracket \times \llbracket 0,2\left(N_{y}-1\right)-1 \rrbracket$ and centered at $\left(N_{x}-1, N_{y}-1\right)$

- use an FFT to calculate $K_{i j}$

- use the translation formula of vector $\left(N_{x}-1, N_{y}-1\right): K_{i j} \leftarrow K_{i j}(-1)^{i+j}$ for $0 \leq i<2(N y-1)$ and $0 \leq j<2\left(N_{x}-1\right)$

or

- extend directly the kernel in a symmetric and periodic kernel defined on $\llbracket 0,2\left(N_{x}-1\right)-1 \rrbracket \times \llbracket 0,2\left(N_{y}-1\right)-1 \rrbracket$. To fix ideas, in 1D the extension of the vector $\left[x_{-2}, x_{-1} x_{0}, x_{1}, x_{2}\right]$ is $\left[x_{0}, x_{1}, x_{2}, 0, \ldots, 0, x_{-2}, x_{-1}\right]$.

- use an FFT to compute $K_{i j}$

Now let us compute the DFT of the dual convolution operator. In $1 \mathrm{D}$, if we denote by $k$ a N-periodic discrete signal associated with a convolution operator, the dual operator is defined by the signal

$$
k^{\star}(i)=k(N-i) \quad \text { for } \quad 1 \leq i<N \quad \text { and } \quad k^{\star}(0)=k(0)
$$

The DFT associated with $K^{\star}$ is then $K_{i}^{\star}=\overline{K_{i}}$. The result generalizes easily in 2D and we deduce that $K_{i j}^{\star}=\overline{K_{i j}}$ (in general $k$ is symmetric and so $K^{\star}=K$ ). To compute solutions of (4.1) and (4.2) we use Algorithm 3

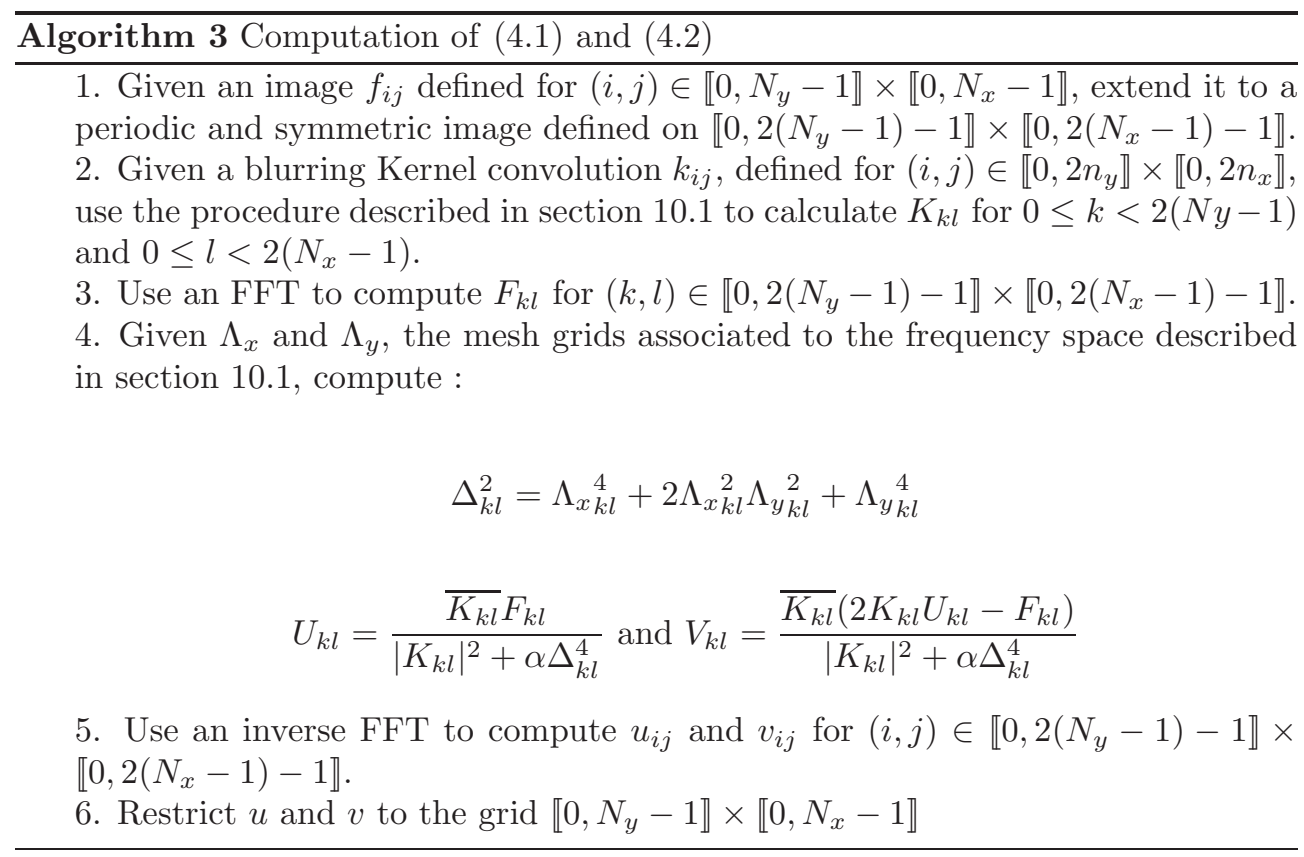

10.2. Numerical results. In this section we test our filaments and points detectors as well the restoration process (5.3) on blurred and noisy images. We separately illustrate the filaments detection given by $I_{\text {Bilap }}^{c}(4.3 \mathrm{~b})$, the filaments and edges restoration (5.3), and the points detection set by (4.3a). 


\section{Filament detection and restoration}

We test two kinds of blurring: the Gaussian blurring and the motion one. A Gaussian blurring is performed by a convolution by a Gaussian of scale $\sigma$ (in our example $\sigma=2.1$ ). This blur can be caused by a bad lense adjustement. It can model a blurring caused by the material. A motion blurring models the effect of the motion of the target or of the camera. It is computed by a convolution with a symmetrical kernel depending on a length parameter (commonly an odd number) and on the angle $\theta$ between the direction of the motion and the horizontal. For more details on the blur origins we refer the reader to [25]. Here the kernel of the motion blur is performed by using the Matlab function $f$ special. On Figure 14 and Figure 15 we compare $I_{\text {Bilap }}^{c}$ (4.3b) and $I_{\text {Hes }}^{f}(6.1)$ on a blurred and noisy image. For the Gaussian blurring, blur effects occur in a similar manner on the whole filament while for the motion blurring, the horizontal part of the filament (perpendicular direction to the motion) is the most degraded. We notice that $I_{\text {Bilap }}^{c}$ still is efficient on blurred and noisy images but that $I_{\text {Hes }}^{f}$ does not allow to detect such degraded filaments.

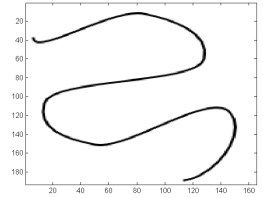

(a) Initial image

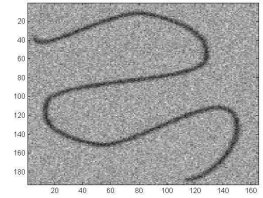

(b) Degraded image

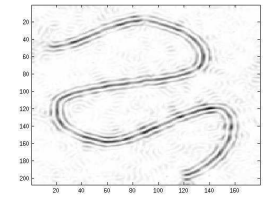

(c) $I_{\text {Bilap }}^{c}(\alpha=0.1)$

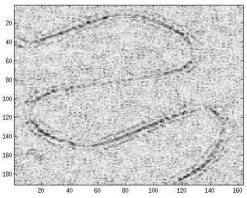

(d) $I_{H e s}^{f}(\sigma=7 / 4)$

FIG. 14. Comparison of $I_{\text {Bilap }}^{c}$ (4.3b) and $I_{H e s}^{f}(6.1)$ for a Gaussian blurred $(\sigma=2.1)$ and noisy $(P S N R=15.7 \mathrm{~dB})$ image

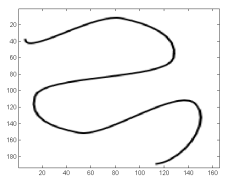

(a) Initial image

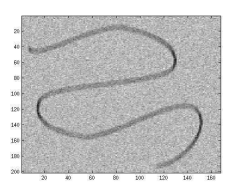

(b) Degraded image

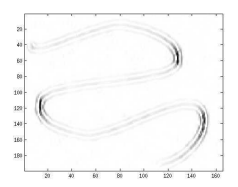

(c) $I_{\text {Bilap }}^{c}(\alpha=0.7)$

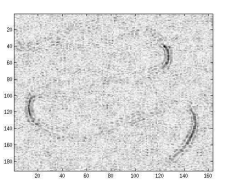

(d) $I_{H e s}^{f}(\sigma=9 / 4)$

FIG. 15. Comparison of $I_{\text {Bilap }}^{c}$ (4.3b) and $I_{\text {Hes }}^{f}$ (6.1) for a motion blurred $\left(\theta=90^{\circ}\right.$, length=7) and noisy image $(P S N R=15.7 d B)$

On Figure 16 and Figure 17, we compare the restoration (5.3) with $\chi_{\eta}=\chi_{\eta}^{\text {Bilap }}$ to a $\mathcal{T V}$ method (see [27]) on respectively a Gaussian blurred and a motion blurred noisy image containing a filament. We see that (5.3) restores very well the filament and the restored version (5.3) is closer to the image to recover than the image obtained by using the $\mathcal{T V}$ method. Particularly, the filament restored by (5.3) is quite well preserved while it is spread out by the $\mathcal{T} \mathcal{V}$ model. Moreover the computational time of (5.3) is shorter than the $\mathcal{T V}$ model (about 5 seconds for (5.3) and 20 seconds for the $\mathcal{T V}$ model for a $170 \times 200$ image).

REMARK 6. We see on Figure 16 that some curved parts of the filament are not very well restored. Indeed if the filament is very curved in the sense of the initial 
resolution of the image, its tangent is not a good aprroximation. Hence, we can first increase the resolution of the image by interpolating the data on the new grid (vectors, image and $I_{\text {Bilap }}^{c}$ ) by using for example bilinear or spline interpolation. Then, it suffices to solve problem (5.3) on the new mesh and then come back to the initial one by an averaging.

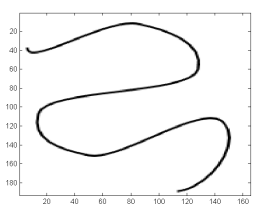

(a) Initial image

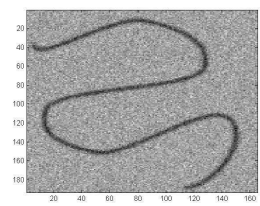

(b) Degraded image

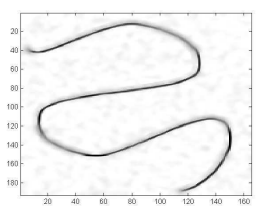

(c) Restored version (d) $\mathcal{T V}$ $(\mathrm{PSNR}=19.8 \mathrm{~dB}) \quad(\mathrm{PSNR}=18.4 \mathrm{~dB})$

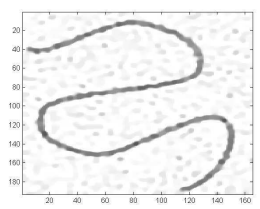

method

FIG. 16. Comparison of the restoration (5.3) $(\beta=1)$ with the $\mathcal{T V}$ model for a Gaussian blurred $(\sigma=1.5)$ and Gaussian noisy $(P S N R=15.7 d B)$ synthetic image

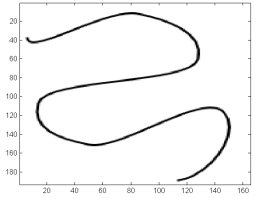

(a) Initial image

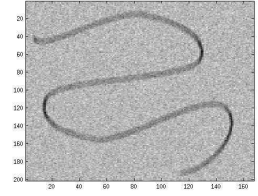

(b) Degraded image
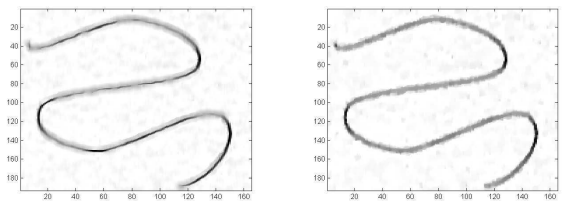

(c) Restored version (d) $\mathcal{T} \mathcal{V}$ method $(\beta=1.2),(\mathrm{PSNR}=20.7(\mathrm{PSNR}=19.4 \mathrm{~dB})$ $\mathrm{dB})$

FIG. 17. Comparison of the restoration (5.3) with the $\mathcal{T} \mathcal{V}$ model for a synthetic image blurred by a convolution of type motion $\left(\theta=90^{\circ}\right.$, length $\left.=7\right)$ and noised by a Gaussian noise (PSNR=15.7dB)

Figure 18 and Figure 19 show the profiles of the image to recover, its degraded versions (blurred and blurred + noisy), the restored version (5.3) with $\chi_{\eta}=\chi_{\eta}^{\text {Bilap }}$ and $I_{\text {Bilap }}^{c}(4.3 \mathrm{~b})$. We see that the restored version (5.3) is close to the image to recover and that $I_{\text {Bilap }}^{c}$ detects the filament. However $I_{\text {Bilap }}^{c}$ has two picks at both side of the filament. We can explain this phenomena as follows. First let us suppose that the continuous image to recover is a Gaussian function that writes as $f(x)=e^{-x^{2} / \eta^{2}}$. We remark that its curvature is maximal at point $x=0$ and it has two local minima at points $x= \pm \sqrt{\frac{3}{2}} \eta$ ( the respective values are $-\frac{2}{\eta^{2}}$ and $\frac{4}{\eta^{2}} e^{-3 / 2}$ ). Then the convolution is implemented by Fourier transform and it is known that the truncation in the Fourier sum generates a aliasing that is increased by the multiplication in the Fourier domain. 


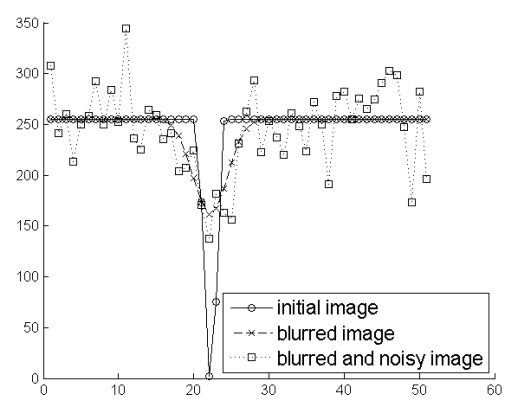

(a)

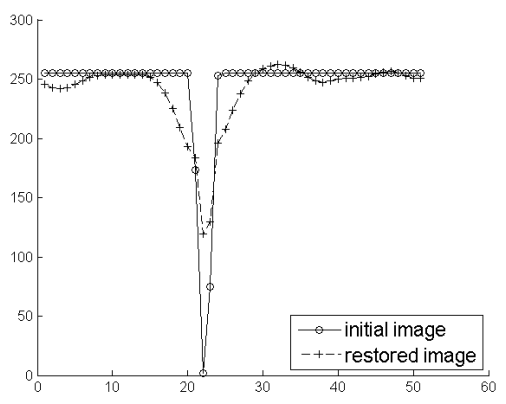

(b)

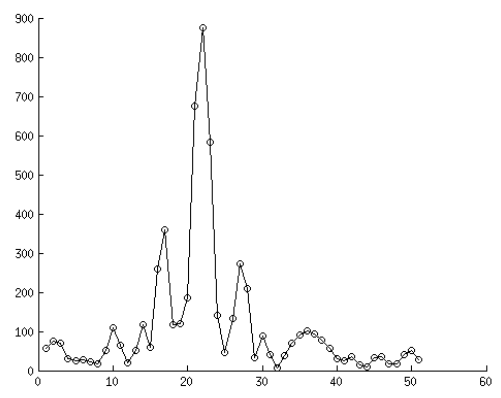

(c)

FIG. 18. (a) A transverse cut displaying the Gaussian blurring $(\sigma=2.1)$ and the Gaussian noise effects $(P S N R=15.7 d B)$, (b) the initial image and the restored version $(5.3)(\beta=1)$ and $(c)$ $I_{\text {Bilap }}^{c}(4.3 \mathrm{~b})$ for $\alpha=0.1$ 


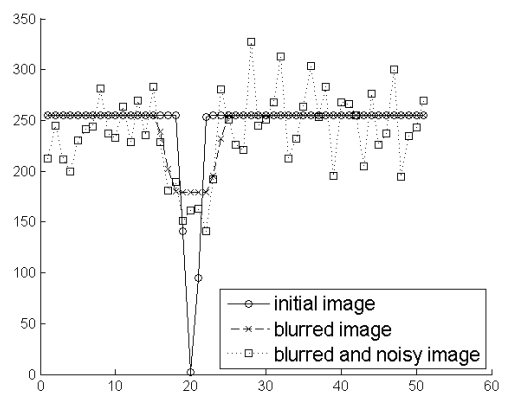

(a)

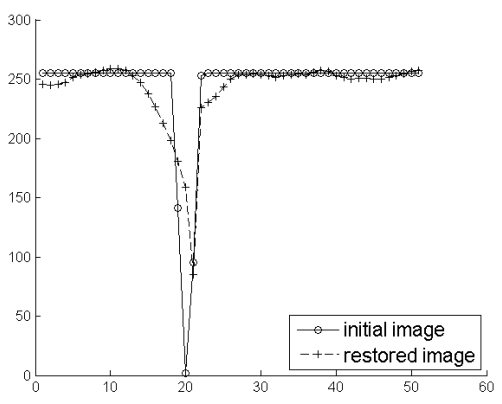

(b)

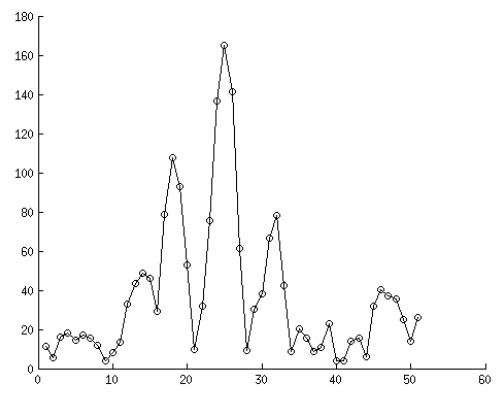

(c)

FIG. 19. (a) A transverse cut displaying the motion blurring $\left(\theta=90^{\circ}\right.$, length=7) and the Gaussian noise effects $(P S N R=15.7 \mathrm{~dB})$, (b) the initial image and the restored version $(5.3)(\gamma=1)$ and (c) $I_{\text {Bilap }}^{c}$ for $\alpha=0.7$

Finally, on Figure 20 we display the restoration (5.3) for different choices of $\chi_{\eta}$ for a Gaussian blurred and noisy image containing both filaments and edges. We see that if we take $\chi_{\eta}=\chi_{\eta}^{\text {Lap }}$ then edges are preserved but filament are spread out.

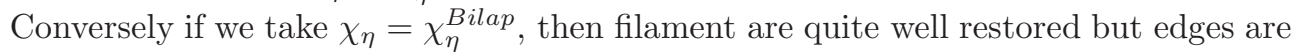
spread out. Finally by taking $\chi_{\eta}=\min \left(\chi_{\eta}^{\text {Bilap }}, \chi_{\eta}^{\text {Lap }}\right)$, then both edges and filaments are well restored. For all experiments the choice of the thresholds $\delta_{1}$ and $\delta_{2}$ defining $\chi_{\eta}$ are such that $3 \%$ of points are in $E_{\delta_{1}}$ and $7 \%$ of points are in $E_{\delta_{2}}$. 


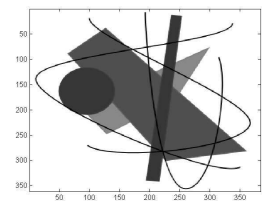

(a) Initial image

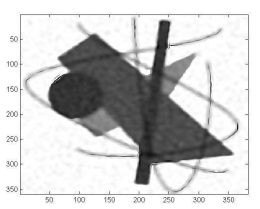

(e) (5.3) $\chi_{\eta}=$ $\min \left(\chi_{\eta}^{\text {Lap }}, \chi^{\text {Bilap }}\right)$ $(\mathrm{PSNR}=20.3 \mathrm{~dB})$

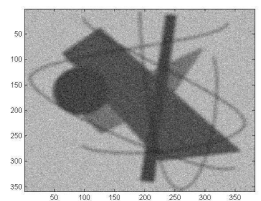

(b) Degraded image

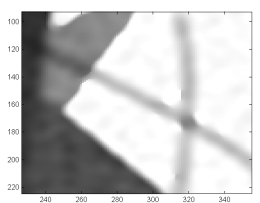

(f) Zoom of (c)
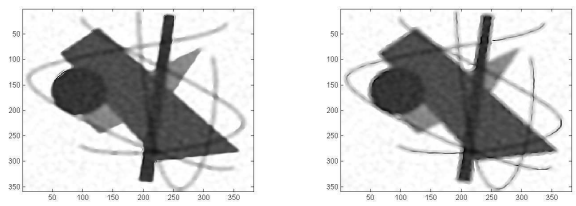

(c) (5.3) $\chi_{\eta}=\chi_{\eta}^{L a p}$ $(\mathrm{PSNR}=19.6 \mathrm{~dB})$

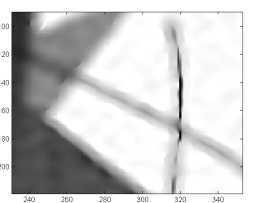

(g) Zoom of (d) (d) (5.3) $\chi_{\eta}=\chi_{\eta}^{\text {Bilap }}$ $(\mathrm{PSNR}=19.6 \mathrm{~dB})$

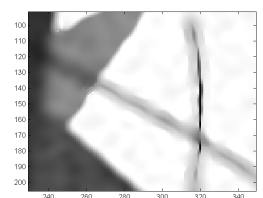

(h) Zoom of (e)

FIG. 20. Comparison of the restoration process (5.3) $(\beta=2)$ with different choices of function $\chi_{\eta}$ for a Gaussian blurred and noisy image $(\sigma=3, P S N R=15.7 d B)$

\section{Point detection}

On Figure 21 and Figure 22 we compare $I_{\text {Bilap }}^{b}(4.3 \mathrm{a})$ to $I_{\text {Hes }}^{p}(6.1)$ on a simple Gaussian noisy and Gaussian blurred image. We see that $I_{\text {Bilap }}^{b}$ detects very well the small structure which is nearly not visible on the degraded image. We see that $I_{\text {Hes }}^{p}$ is not efficient on this type of image.

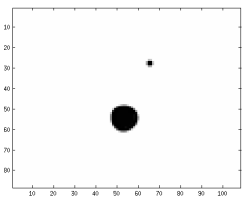

(a) Initial Image

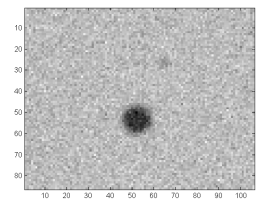

(b) Degraded image

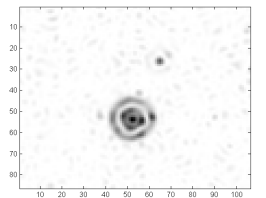

(c) $I_{\text {Bilap }}^{b}(\alpha=1.2)$

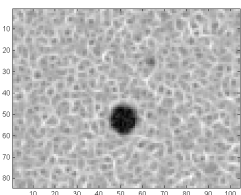

(d) $I_{H e s}(\sigma=5 / 4)$

FIG. 21. Points detection by the topological indicator $I_{\text {Bilap }}^{b}(4.3 \mathrm{a})$ and the indicator $I_{\text {Hes }}(6.1)$ on a synthetic image blurred by a Gaussian convolution $(\sigma=1.5)$ and noised by a Gaussian noise $(P S N R=15.7 d B)$ 


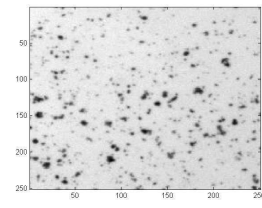

(a) Initial real image

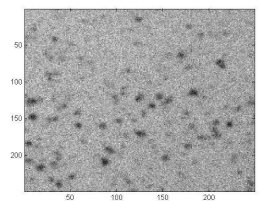

(b) Degraded image

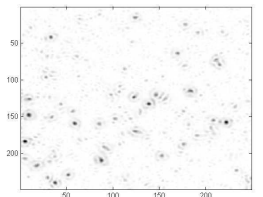

(c) $I_{\text {Bilap }}^{b},(\beta=1.2)$

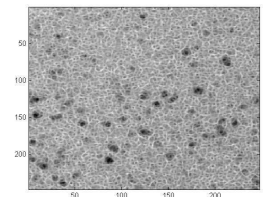

(d) $I_{H e s},(\sigma=5 / 4)$

FIG. 22. Points detection by the topological indicator $I_{\text {Bilap }}^{b}$ and the indicator $I_{H e s}$ on a synthetic image blurred by a Gaussian convolution $(\sigma=1.5)$ and noised by a Gaussian noise $(P S N R=18 d B)$

11. Application to a 3D image. In [13], the topological gradient of (2.1) for the problem (2.5) is explicitly given in 2D. In $3 \mathrm{D}, B_{1}$ and $B_{2}$ for $\nu=0$ generalize as

$$
\begin{aligned}
& B_{2}(u)=\frac{\partial^{2} u}{\partial x_{i} \partial x_{j}} n_{i} n_{j} \\
& B_{1}(u)=\operatorname{div}_{s}\left(\frac{\partial^{2} u}{\partial x_{i} \partial x_{j}} n_{i} \overrightarrow{e_{j}}\right)+\frac{\partial^{3} u}{\partial x_{i}^{2} \partial x_{j}} n_{j}
\end{aligned}
$$

where we use the repeated subscript notation and with $d i v_{s}$ is the surface divergence operator defined by $\operatorname{div}_{s}(\vec{q})=P: \operatorname{Jac}(\vec{q}) . P$ is the projector $P_{i j}=\left(\delta_{i j}-n_{i} n_{j}\right)$ and $\operatorname{Jac}(\vec{q})$ the Jacobian matrix of $\vec{q}(x) . A: B$ denotes the scalar product of matrices A and $\mathrm{B}$. Then the only change (in comparison with the $2 \mathrm{D}$ case) is the scaling parameter factor in the topological gradient expression which is $\epsilon^{3}$ (instead $\epsilon^{2}$ in 2D). For more details we refer the reader to [12]. We define the following planar circular crack

$$
\sigma=\{(r \cos (\theta), r \sin (\theta), 0), 0 \leq r<1,0 \leq \theta<2 \pi\} .
$$

Then a crack of normal $\vec{n}=(\sin (\varphi) \cos (\xi), \sin (\varphi) \sin (\xi), \cos (\varphi))^{T}$ is deduced from this definition by writing the same thing in the orthonormal basis $(\vec{v}, \vec{w}, \vec{n})$ with $\vec{v}=$ $(-\sin (\xi), \cos (\xi), 0)^{T}$ and $\vec{w}=(-\cos (\varphi) \cos (\xi),-\cos (\varphi) \sin (\xi), \sin (\varphi))^{T}$ for $0 \leq \varphi<\pi$ and $0 \leq \xi<2 \pi$. Thus, the topological gradient for a cracked domain in 3D is (see $[12]):$

$$
I_{\text {Bilap }}^{c}\left(x_{0}\right)=\max _{\|\vec{n}\|=1}\left|\mathcal{I}_{c}\left(x_{0}, \vec{n}\right)\right|
$$

where

$$
\mathcal{I}_{c}\left(x_{0}, \vec{n}\right)=-\frac{4 \pi}{3} \nabla^{2} u_{0}\left(x_{0}\right)(\vec{n}, \vec{n}) \nabla^{2} v_{0}\left(x_{0}\right)(\vec{n}, \vec{n})
$$

In this section, in order to shorten notations, we still denote by $I_{\text {Bilap }}^{c}$ (11.1) the bilaplacian topological gradient for a cracked domain in 3D. On Figure 23 we display $I_{\text {Bilap }}^{c}$ on two noisy images. We precise that results displayed here are obtained by using the 3D DCT and the 3D inverse DCT in Algorithm 1 (see section 7.2). Initial images contain two kinds of structures : filament of dimension 1 in 3D (modeled by a cylinder of radius 3 pixels of axis the z-axis) and surfaces of dimension 2 in $3 \mathrm{D}$ (modeled by two concentric spheres of width 4 or 5 pixels). We denote by $\left(x_{c}, y_{c}, z_{c}\right)$ the coordinates of the center of the spheres. Figure 24 shows a $2 \mathrm{D}$ cut in the plane $z=z_{c}$ of the initial images and of $I_{\text {Bilap }}^{c}$ and Figure 25 is a $1 \mathrm{D}$ cut in the line $z=z_{c}$ 
and $y=y_{c}$. We see that surfaces and filaments are well detected by $I_{\text {Bilap }}^{c}(11.1)$ in $3 \mathrm{D}$.

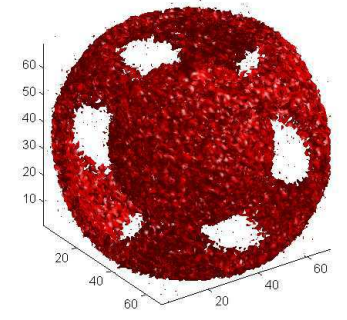

(a) Noisy image

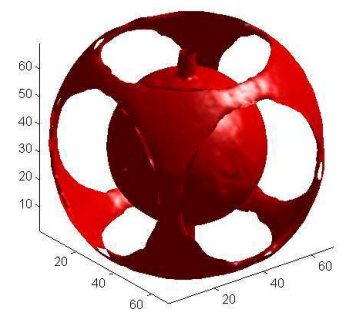

(c) Noisy image

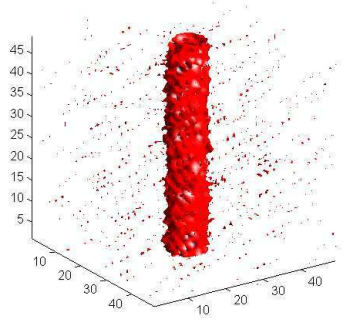

(b) $I_{\text {Bilap }}^{c}(11.1)$

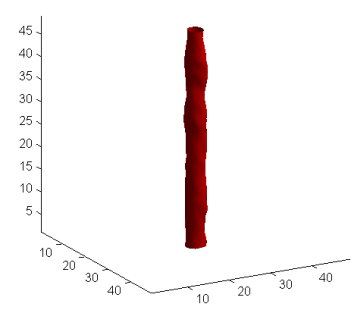

(d) $I_{\text {Bilap }}^{c}(11.1)$

FIG. 23. Isosurfaces of $I_{\text {Bilap }}^{c}$ (11.1) for two Gaussian noisy images (PSNR=16dB)

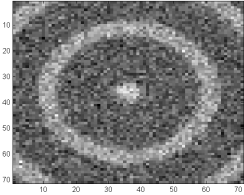

(a) Noisy image

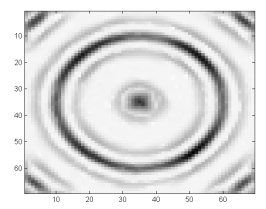

(b) $I_{\text {Bilap }}^{c}(\alpha=0.25)$

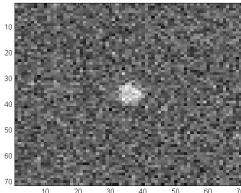

(c) Noisy image

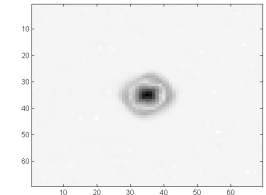

(d) $I_{\text {Bilap }}^{c}(\alpha=0.25)$

FIG. 24. A 2D cut $\left(z=z_{c}\right)$ of $I_{\text {Bilap }}^{c}$ (11.1) for two Gaussian noisy images (PSNR=16dB).

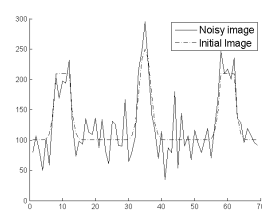

(a) Noisy image

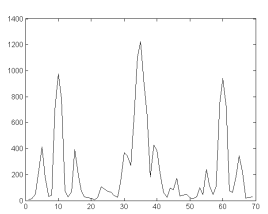

(b) $I_{\text {Bilap }}^{c}(\alpha=100)$

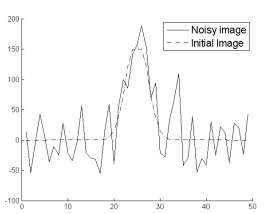

(c) Noisy image

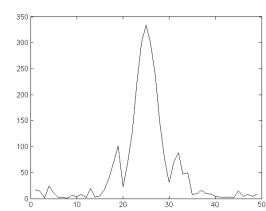

(d) $I_{\text {Bilap }}^{c}(\alpha=400)$

FIG. 25. A $1 D$ cut $\left(y=y_{c}\right)$ and $\left(z=z_{c}\right)$ of $I_{\text {Bilap }}^{c}$ (11.1) for two Gaussian noisy images $(P S N R=16 d B)$. 
REMARK 7. We can show that $I_{\text {Bilap }}^{c}$ (11.1) detects also points in 3D (modeled by small spheres). But, the topological indicator for a perforated domain seems more adapted to do this. Its computation is very technical and it will be done in future works.

\section{REFERENCES}

[1] J.P. Antoine, R. Murenzi, And P. Vandergheynst, Directional Wavelets Revisited: Cauchy Wavelets and Symmetry Detection in Patterns, Applied and Computational Harmonic Analysis, 6 (1999), pp. 314-345.

[2] G. Aubert, J.F Aujol, and L. Blanc-Féraud, Detecting Codimension - Two Objects in an Image with Ginzburg-Landau Models., 65 (2005), pp. 29-42.

[3] G. Aubert and P. Kornprobst, Mathematical Problems in Image Processing: Partial Differential Equations and the Calculus of Variations (second edition), vol. 147 of Applied Mathematical Sciences, Springer-Verlag, 2006.

[4] D. Auroux, From restoration by topological gradient to medical image segmentation via an asymptotic expansion, Math. Comput. Model., 49 (2009), pp. 2191-2205.

[5] D. Auroux, M. Masmoudi, and L. JaAfar Belaid, Image restoration and classification by topological asymptotic expansion, Variational Formulations in Mechanics: Theory and Applications, E. Taroco, E.A. de Souza Neto and A.A. Novotny (Eds), CIMNE, Barcelona, Spain, 2007, pp. 23-42.

[6] A. BAudour, Détections de Filaments dans des images 2D et 3D; modélisation, étude mathématique et algorithmes, Phd Dissertation, Université de Nice Sophia Antipolis, (2009).

[7] A. Baudour, G. Aubert, and L. Blanc-Féraud, Detection and Completion of Filaments: A Vector Field and PDE Approach., in SSVM, Fiorella Sgallari, Almerico Murli, and Nikos Paragios, eds., vol. 4485 of Lecture Notes in Computer Science, Springer, 2007, pp. 451-460.

[8] L. Jahafar Belaid, M. Jaoua, M. Masmoudi, and L. Siala, Application of the topological gradient to image restoration and edge detection, Engineering Analysis with Boundary Elements, 32 (2008), pp. $891-899$.

[9] S. Berlemont and J-C. Olivo-Marin, Combining Local Filtering and Multiscale Analysis for Edge, Ridge, and Curvilinear Objects Detection., IEEE Transactions on Image Processing, 19 (2010), pp. 74-84.

[10] S.C. Brenner and L.R. Scott, The Mathematical Theory of Finite Element Methods, Springer-Verlag, 2002.

[11] J. Shah D. Mumford, Optimal approximations by piecewise smooth functions and associated variational problems, Communications on Pure and Applied Mathematics, 42 (1989), pp. 577-685.

[12] A. Drogoul, Applications of the topological gradient in the detection of fine structures in imaging, Phd Thesis University of Nice Sophia Antipolis, (2014).

[13] A. Drogoul and G. Aubert, Topological gradient for fourth order PDE and application to the detection of fine structures in 2D images (submitted), Control, Optimization and Calculus of Variations.

[14] A. Drogoul and G. Aubert, The topological gradient method for semi-linear problems and application to edge detection and noise removal (submitted), Journal of Mathematical Imaging and Vision, (2014).

[15] J. Zhou G. Chen, Boundary Element Methods with Applications to Nonlinear Problems, Artlantis studies in Mathematics for Engineering and Science, 1992.

[16] D. Graziani, G. Aubert, and L. Blanc-Féraud, A formal Gamma-convergence approach for the detection of points in 2-D biological images, SIAM Journal on Imaging Sciences, 3 (2010), pp. 578-594.

[17] S.G. Johnson, Notes on fft-based differentiation, MIT Applied Mathematics, (2011).

[18] C. Lacoste, X. Descombes, and J. Zerubia, Point Processes for Unsupervised Line Network Extraction in Remote Sensing, IEEE Transactions on Pattern Analysis and Machine Intelligence, 27 (2005), pp. 1568-1579.

[19] KM. Liew And Q. Wang, Application of Wavelet Theory for Crack Identification in Structures., Journal of Engineering Mechanics, 124 (1998), pp. 152-157.

[20] M. Jacob and T. Blu and C. Vaillant and J.H. Maddocks and M. Unser, $3 D$ shape estimation of DNA molecules from stereo cryo-electron micro-graphs using a projectionsteerable snake, IEEE Trans. Image Process, 15 (2006), pp. 214-227. 
[21] M. Masmoudi, The topological asymptotic, in Computational Methods for Control Applications, vol. 16 of GAKUTO Internat. Ser. Math. Appl., Tokyo, Japan, 2001.

[22] A.M Mendona And A.C. CAmpilho, Segmentation of retinal blood vessels by combining the detection of centerlines and morphological reconstruction., IEEE Trans. Med. Imaging, 25 (2006), pp. 1200-1213.

[23] W. Ming AND J. XU, Nonconforming tetrahedral finite elements for fourth order elliptic equations., Math. Comput., 76 (2007), pp. 1-18.

[24] C. Cañero Morales and P. Radeva, Vesselness enhancement diffusion, Pattern Recognition Letters, 24 (2003), pp. 3141-3151.

[25] J. PAWley, Handbook of Biological Confocal Microscopy, Springer, Berlin, 2006.

[26] M. Rochery, I.H. Jermyn, And J. Zerubia, New higher-order active contour energies for network extraction., in ICIP (2), IEEE, 2005, pp. 822-825.

[27] L.I. Rudin, S. Osher, ANd E. FATEmi, Nonlinear total variation based noise removal algorithms, Phys. D, 60 (1992), pp. 259-268.

[28] M. Masmoudi S. Amstutz, I. Horchani, Crack detection by the toplogical gradient method, Control and Cybernetics, 34 (2005), pp. 81-101.

[29] J. Sokolowski AND A. Zochowski, On the topological derivative in shape optimization, SIAM J. Control Optim., 37 (1999), pp. 1251-1272.

[30] C. Steger, An unbiased detector of curvilinear structures, IEEE Trans. Pattern Anal. Mach. Intell., 20 (1998), pp. 113-125.

[31] C. Steger, Estimating the fundamental matrix under pure translation and radial distortion, ISPRS Journal of Photogrammetry and Remote Sensing, 74 (2012), pp. 202-217.

[32] R. Stoica, X. Descombes, and J. Zerubia, A Gibbs Point Process for Road Extraction from Remotely Sensed Images., International Journal of Computer Vision, 57 (2004), pp. 121136.

[33] F. Tupin, J.F. Mangin, E. Pechersky, J.M. Nicolas, and H. Maître, A Graph-Based Representation to Detect Linear Features, 12 (1998), pp. 21-31.

[34] F. ZANA AND J-C. KLEIN, Segmentation of vessel-like patterns using mathematical morphology and curvature evaluation., IEEE Transactions on Image Processing, 10 (2001), pp. 10101019. 